DR. CARMELO ANDUJAR (Orcid ID : 0000-0001-9759-7402)

PROF. ALFRIED VOGLER (Orcid ID : 0000-0002-2462-3718)

Article type : Original Article

\title{
Metabarcoding of freshwater invertebrates to detect the effects of a pesticide spill
}

\section{Carmelo Andújar ${ }^{1,2,3}$, Paula Arribas ${ }^{1,2,3}$, Clare Gray ${ }^{2}$, Katherine Bruce ${ }^{4}$, Guy Woodward ${ }^{2}$,} Douglas W. Yu ${ }^{5,6}$, Alfried P. Vogler ${ }^{1,2}$

${ }^{1}$ Department of Life Sciences, Natural History Museum, Cromwell Road, London, SW7 5BD, UK

${ }^{2}$ Department of Life Sciences, Imperial College London, Silwood Park Campus, Ascot, SL5 7PY, UK

${ }^{3}$ Grupo de Ecología y Evolución en Islas, Instituto de Productos Naturales y Agrobiología (IPNA-CSIC), San Cristóbal de la Laguna 38206, Spain

${ }^{4}$ NatureMetrics Ltd, CABI Site, Bakeham Lane, Egham, Surrey, TW20 9TY, UK

${ }^{5}$ State Key Laboratory of Genetic Resources and Evolution, Kunming Institute of Zoology, Chinese

Academy of Sciences, Kunming, Yunnan 650223, China

${ }^{6}$ School of Biological Sciences, University of East Anglia, Norwich Research Park, Norwich, Norfolk NR47TJ, UK

Correspondence to:

Dr Carmelo Andujar, Grupo de Ecología y Evolución en Islas, Instituto de Productos Naturales y

Agrobiología (IPNA-CSIC), San Cristóbal de la Laguna 38206, Spain

Email candujar@um.es

Running title: Metabarcoding of freshwater invertebrates

This article has been accepted for publication and undergone full peer review but has not been through the copyediting, typesetting, pagination and proofreading process, which may lead to differences between this version and the Version of Record. Please cite this article as doi: $10.1111 / \mathrm{mec} .14410$

This article is protected by copyright. All rights reserved. 


\begin{abstract}
Biomonitoring underpins the environmental assessment of freshwater ecosystems and guides management and conservation. Current methodology for surveys of (macro)invertebrates uses coarse taxonomic identification where species-level resolution is difficult to obtain. Next-generation sequencing of entire assemblages (metabarcoding) provides a new approach for species detection, but requires further validation. We used metabarcoding of invertebrate assemblages with two fragments of the coxl "barcode" and partial nuclear ribosomal ( $S S U$ ) genes, to assess the effects of a pesticide spill in the River Kennet (Southern England). Operational Taxonomic Unit (OTU) recovery was tested under 72 parameters (read denoising, filtering, pair merging and clustering). Similar taxonomic profiles were obtained under a broad range of parameters. The SSU marker recovered Platyhelminthes and Nematoda, missed by coxl, while Rotifera were only amplified with coxl. A reference set was created from all available barcode entries for Arthropoda in the BOLD database and clustered into OTUs. The River Kennet metabarcoding produced matches to 207 of these reference OTUs, five times the number of species recognised with morphological monitoring. The increase was due to: greater taxonomic resolution (e.g. splitting a single morphotaxon 'Chironomidae' into 55 named OTUs); splitting of Linnaean binomials into multiple molecular OTUs; and the use of a filtrationflotation protocol for extraction of minute specimens (meiofauna). Community analyses revealed strong differences between "impacted" vs. "control" samples, detectable with each gene marker, for each major taxonomic group, and for meio- and macro-faunal samples separately. Thus, highly resolved taxonomic data can be extracted at a fraction of the time and cost of traditional nonmolecular methods, opening new avenues for freshwater invertebrate biodiversity monitoring and molecular ecology.
\end{abstract}

\title{
INTRODUCTION
}

The freshwater biota is affected by a host of natural environmental drivers and, increasingly, anthropogenic disturbances that alter local species assemblages. Biomonitoring therefore is required to assess the ecological status of freshwaters and to enforce their protection through legislation, such as the Water Framework Directive (WFD) of the European Union and the US Clean Water Act (United States 1972; European Commission 2000). However, this field of applied ecology is still largely reliant on techniques that were developed over a century ago, albeit with some statistical advances, tweaks, and adjustments in the intervening years, and has been roundly criticised for failing to adapt to a rapidly changing world (Friberg et al. 2011). The vast majority of biomonitoring schemes still relies on identifying macroinvertebrates by eye, or at best via microscopy, to a coarse level of taxonomic resolution, and the molecular revolution that is overtaking mainstream ecology has yet to be embraced (Pauls et al. 2014; Bohan et al. 2017). Because of the need for rapid and cost-

This article is protected by copyright. All rights reserved. 
effective approaches, it is routine practice that many taxa are not identified to individual species but instead are lumped taxonomically, e.g. by family, as used in RIVPACS and AUSIVAS systems (Wright et al. 2000), or, less frequently, into trait-based groupings, such as "riverflies". The taxonomically difficult groups in which most of the aquatic biodiversity resides (e.g. chironomid midges) are typically either ignored or treated as a single entity (Schmidt-Kloiber \& Nijboer 2004; Jones 2008).

These labour-saving shortcuts can nonetheless provide a broad assessment of the ecological state of a water body, despite the huge amounts of environmental-status information that are inevitably jettisoned in the process, and has been successfully used for the assessment of habitat and water quality for many decades (Camargo 1993; Marshall et al. 2006; Sánchez-Montoya et al. 2007). However, population responses to changes in water quality can differ between even closely related species, and so taxonomically coarse inventories may miss the full impact of important environmental stressors (Stubauer \& Moog 2000; Chessman et al. 2002; Gutiérrez-Cánovas et al. 2008). Specieslevel identification can establish the link to known ecological, physiological and behavioural traits, which may reflect differential responses to environmental conditions, and also may reveal the membership in feeding groups and position in trophic networks (Bohan et al. 2017). These distinctions are lost if the assessment is at the level of genera or families, or other such coarse groupings (Schmidt-Kloiber \& Hering 2015; Leese et al. 2016).

Recent protocols for metabarcoding, i.e. the sequencing of PCR amplicons from environmental specimen mixtures, could provide faster and more highly-resolved taxonomic identification of complex assemblages (Taberlet et al. 2012). This methodology applies Hebert et al.'s (2003) idea of species identification through short diagnostic DNA barcodes (a fragment of the coxl gene) to the community level, and thanks to new high-throughput sequencing (HTS) technology, the effort required for DNA barcoding of an entire assemblage now is not much greater than required for a single specimen with Sanger-sequencing (Taylor \& Harris 2012; Brandon-Mong et al. 2015). Metabarcoding permits the simultaneous analysis of large numbers of minute specimens obtained from environmental samples, such as soil and leaf litter (Yang et al. 2014; Arribas et al. 2016; Zinger et al. 2016), the deep sea (Esling et al. 2015; Guardiola et al. 2015; Leray \& Knowlton 2015; Lanzén et al. 2016), and freshwater sediments and the water column (Elbrecht \& Leese 2015; Bista et al. 2017). Metabarcoding can thus provide the elusive species-resolution desired for biomonitoring of entire ecosystems, and also for capturing the large proportion of organisms that are either too small to see or identify using traditional sorting and microscopy techniques (Creer et al. 2010; Ji et al. 2013; Hajibabaei et al. 2016; Bohan et al. 2017).

Here, we applied metabarcoding to study the consequences of an insecticide spill on invertebrate freshwater communities in a large lowland river as a test case. On July 1, 2013, a pulse of the

This article is protected by copyright. All rights reserved. 
organophosphate chlorpyrifos in the River Kennet, the largest tributary of the River Thames in southern England, led to population crashes and localised extinctions of many invertebrate taxa (see Thompson et al. 2016 for details). We used samples collected upstream and downstream from the spill site over several km of the river's length to explore the effectiveness of metabarcoding, and to trial new environmental diagnostic protocols for identifying differential responses of invertebrate communities to a profound environmental perturbation. For example, some components of the local community such as the dominant detritivore, the amphipod Gammarus pulex, were greatly reduced in number downstream from the spill, whereas other taxa, especially those with an aerial adult life stage, were far less affected, and at a later sampling time returned to post-spill levels, possibly due to their ability to recolonize rapidly. The Chironomidae (non-biting midges) as a group greatly increased in abundance after the spill. However, because community composition was only measured at higher taxonomic levels, rather than with species-level resolution, it is not possible to gain further insight into the mechanisms of ecological resilience and recovery after the spill. Specifically, the species composition of the post-impact chironomid community might be largely unchanged from the preimpact community, or, despite the increased abundance, it might be composed of a subset of that community (nestedness) or of a new set of species dispersed from elsewhere (turnover).

The coxl gene is the obvious choice of a marker for metabarcoding of aquatic invertebrates, but due to the constraints on read length, the widely used Illumina platform is not suited for sequencing the full-length amplicon of the barcode region (658 bp). We have metabarcoded two gene fragments covering the entire coxl barcode region using two primer pairs shown to have broad target ranges (Arribas et al. 2016), which here were applied for the first time to freshwater invertebrate communities. The parallel use of two barcode fragments provides a test of amplification breadth and potential biases due to primer choice, which could affect the success of species detection and delimitation. A major concern is that PCR amplification of the coxl region in several aquatic phyla is generally low and thus this region may produce bias in the detectable species assemblages (Deagle et al. 2014; Lobo et al. 2015; Creer et al. 2016). We therefore also conducted metabarcoding with the nuclear 18S rRNA ( $S S U$ ) gene, frequently used for sequencing marine meiofaunal communities but never tested in freshwater ecosystems to our knowledge. This gene contains highly conserved regions bracketing more variable segments and thus is less affected by primer bias across a larger phylogenetic range of taxa. However, lower sequence variation in $S S U$ generally underestimates the true species diversity (Tang et al. 2012). The resulting metabarcode sequences are typically first clustered into de novo generated species proxies, i.e. Operational Taxonomic Units (OTUs) (Blaxter et al. 2005), that can be directly used for downstream ecological analyses. Species identification is critical for many uses of these data, and can be obtained against existing databases of DNA sequences from fully identified specimens available at public databases (NCBI or BOLD). These reference sets can be used in two ways, either by matching the de novo generated OTUs against the external

This article is protected by copyright. All rights reserved. 
reference sequences, in a 'taxonomy independent' approach, or by matching the raw sequence reads directly to the reference set without prior OTU clustering in a 'taxonomy dependent' approach (Schloss \& Westcott 2011), which has been used on various occasions to test species presence or absence (e.g. Shokralla et al. 2014; Arribas et al. 2016).

The River Kennet pesticide spill, characterised previously with conventional approaches (Thompson et al. 2016), was used to trial the metabarcoding methodology for freshwater invertebrates. This included the development of protocols for extraction of meio- and macro-fauna from bulk sediment samples, the evaluation of existing universal primers for amplification of the coxl and nuclear $18 \mathrm{~S}$ ribosomal RNA ( $S S U$ ) genes, and the calibration of bioinformatics tools and parameter settings for accurate estimates of species numbers and species identification. For identification of the local community we made use of the rapidly growing publicly available taxonomic sequence databases, whose species representation is increasingly complete at least for this ecosystem in Western Europe. Given the high quality of sequence data achievable with recent Illumina technology, future biomonitoring schemes may shift to the use of metabarcoding.

\section{MATERIALS AND METHODS}

\section{Study site and sampling protocol}

The River Kennet is a lowland chalk river that was affected by widespread macroinvertebrate mortality along a 15-km stretch downstream from an insecticide spill site (Thompson et al. 2016). Invertebrates were collected using a Surber sampler $\left(0.0625 \mathrm{~m}^{2}, 335 \mu \mathrm{m}\right.$ mesh $)$ at three upstream control and three downstream impacted reaches, each $50 \mathrm{~m}$ long, along a ca. $6 \mathrm{~km}$ river stretch (including the four sites sampled in Thompson et al. 2016). Sites were ca. $1 \mathrm{~km}$ apart, with similar channel forms and riparian surroundings, and were sampled at two times: time 1 (12th July 2013), 11 days after the spill; time 2 (17th September 2013) (Suppl. Fig. S1). The latter was the same time as samples used in Thompson et al. (2016). The sampling regime permitted to explore the immediate effect downstream of the spill point relative to the unaffected upstream sites, and the short-term recovery of the arthropod community 2.5 months after the spill. One Surber sample per site and time was preserved immediately after collection in absolute ethanol and transferred to the laboratory, where we removed debris by hand and subsequently filtered the remainder through a $1 \mathrm{~mm}$ wire mesh sieve to retain macrofauna. The smaller material not retained by this sieve was then passed through a $45 \mu \mathrm{m}$ wire mesh sieve to capture the meiofaunal fraction (size $<1 \mathrm{~mm}$ ) while flushing out microorganisms and silt with copious amounts of water (Fonseca et al. 2010; Arribas et al. 2016). Note that the original Surber used a $335 \mu \mathrm{m}$ mesh but many organisms below this size were retained in the Surber sample by debris and no effort was made to remove small organisms at this stage. The

This article is protected by copyright. All rights reserved. 
filtrate from this second step was cleaned by flotation using LUDOX $40^{\mathrm{TM}}$ (Burgess 2001) to separate organisms, which tend to float, from inorganic particles, which tend to sink. The floating layer was extracted for DNA to represent the sampled meiofauna. Each sample was processed separately for the macro- and meiofauna, for a final number of 24 samples used for DNA extraction and sequencing (Fig. 1).

\section{DNA extraction and Illumina sequencing}

Each sample was dried and homogenised in a Falcon tube, and DNA was extracted from $200 \mu 1$ of sample lysate using a DNeasy Blood and Tissue Spin-Column Kit (Qiagen). Three DNA markers were individually amplified: a fragment of the $S S U$ gene, and two fragments ( $b c 5^{\prime}$ and $\left.b c 3^{\prime}\right)$ within the mitochondrial coxl barcode region. The two fragments were bracketed by the "Folmer" primers used for amplification of the standard animal barcode (Hebert et al., 2003), but with a higher degree of degeneracy. The $b c 5^{\prime}$ fragment corresponds to $\approx 350 \mathrm{bp}$ of the 5 ' end of the coxl barcode fragment, and was amplified using primers already validated in a wide variety of arthropods (Fol-degen-for: 5' TCNACNAAYCAYAARRAYATYGG (Yu et al. 2012) and Ill_C_R: 5'

GGIGGRTAIACIGTTCAICC (Shokralla et al. 2015). Similarly, the $b c 3^{\prime}$ fragment corresponding to $\approx 420$ bp of the 3 ' end of the coxl barcode was amplified with primers Ill_B_F (5'-

CCIGAYATRGCITTYCCICG) (Shokralla et al. 2015) and Fol-degen-rev (5'-

TANACYTCNGGRTGNCCRAARAAYCA) (Yu et al. 2012). The $S S U$ marker was amplified using primers SSU-FO4 (5'-GCTTGTCTCAAAGATTAAGCC) and SSU-R22 (5'-

GCCTGCTGCCTTCCTTGGA), producing a fragment of varying length of 300 to $400 \mathrm{bp}$ (Blaxter $e t$ al. 1998).

Primers were modified to include an overhang adapter sequence for subsequent nested PCR, in analogy to the Illumina protocol for sequencing the 16S rRNA gene in microbial samples (16S Library Preparation Protocol at http://support.illumina.com) (see Arribas et al. 2016). For each sample, three independent reactions for each pair of primers were performed, and the PCR amplicons were pooled. All information regarding PCR reagents and conditions was included in Data S1.

Amplicon pools were cleaned using Ampure XP magnetic beads, after which these primary amplicons were used as template for a limited-cycle secondary PCR amplification to add dual-index barcodes and the P5 and P7 Illumina sequencing adapters (Nextera XT Index Kit; Illumina, San Diego, CA, USA). For each sample, the three gene fragments were processed individually but using the same indexes for sample tagging, thus combined in a single library and reducing the costs. The 24 resulting metabarcoding libraries were sequenced on an Illumina MiSeq sequencer (2x300 bp paired end reads) on $1.5 \%$ of the flow cell each, to produce paired reads (R1 and R2) with a given dual tag unique combination for each sample.

This article is protected by copyright. All rights reserved. 


\section{Creating a reference sequence database}

A custom reference set of OTUs for Arthropoda was created from sequences obtained from the BOLD Public Data Portal (Ratnasingham \& Hebert 2007; accessed on 8th January 2017). All available fulllength (658 bp) coxl sequences for Arthropoda were retrieved from BOLD and subsequently clustered with Usearch v7 (Edgar 2013) under a 3\% similarity threshold. This resulted in thousands of OTUs (referred to as BOLD-OTUs from hereon), each of which was based on variable numbers of primary entries in the BOLD database, ranging from just a single sequence to several hundred sequences in some cases. For simplicity, the centroid sequence of each BOLD-OTU was used as the "representative sequence" in subsequent analyses for the taxonomic identification of metabarcoding sequences. The BOLD database already provides clustering of barcode data based on a graph theory method that produces the so-called BIN (Barcode Identification Number) groups (Ratnasingham \& Hebert 2013). We established the correspondence of our BOLD-OTUs with the BINs based on the representative sequences, which permitted to attach a BIN number and, where available, the associated species name to the BOLD-OTUs. We obtained species names for most of the OTUs that were matched by the metabarcoding study, but there were 18 cases where metabarcoding reads matched a single sequence on BOLD that was not attached to any named BIN group and which was identified to order level only.

In general, each $B O L D-O T U$ corresponded to a unique Linnaean species name, but in several cases the same species name was attached to multiple BOLD-OTUs, indicating high intraspecific genetic diversity, identification problems in the reference database, or the existence of cryptic species (Table 1, Suppl. Table 4). The BINs on the BOLD database equally are affected by splitting of Linnaean species. For example, sequences associated with the isopod Asellus aquaticus were assigned to eight separate BOLD-OTUs matching 8 different BINs. High intraspecific variation ( $>3 \%$ divergence in coxl) is a well-established observation in the case of Asellus (Sworobowicz et al. 2015). Three BOLD-OTUs were assigned to Baetis rhodani (Ephemeroptera), which is also reflected in the incomplete taxonomy of this species complex (Williams et al. 2006; Bisconti et al. 2016).

\section{Bioinformatic read processing}

Various bioinformatics steps were applied to reduce the proportion of low-quality data (Schirmer et al. 2015). These steps included the trimming of 3' ends, merging $\mathrm{R} 1$ and $\mathrm{R} 2$ reads, and the detection and removal of hybrid molecules formed during PCR from mixed templates. Raw reads were quality checked in Fastqc (Babraham Institute 2013) and subsequently de-multiplexed to get independent datasets for each of the three DNA fragments, using the fastx_barcode_splitter.pl option of the FASTX-Toolkit (http://hannonlab.cshl.edu/fastx_toolkit/; accessed 18/07/2016). Primers were

This article is protected by copyright. All rights reserved. 
trimmed using fastx_trimmer and reads were processed in Trimmomatic (Bolger et al. 2014) using TRAILING:20 MINLEN:250 CROP:270 (CROP 250 for R2). R1 and R2 reads were paired using Pairfq 0.16 (Staton 2013) and the makepairs option.

Alternative procedures for read denoising, pair merging, quality filtering and clustering method were tested for each DNA marker (Suppl. Fig. S2). These analyses included: (i) Four different denoising parameters using BFC ( $\mathrm{Li} 2015$ ) ( $\mathrm{s}=0.35 \mathrm{~K}=33$; $\mathrm{s}=2 \mathrm{~K}=33$; $\mathrm{s}=20 \mathrm{~K}=33$; without de-noising); (ii) two procedures for pair merging using either Pear v0.9.6 (Zhang et al. 2014) with $-q 26$ and other default parameters or Usearch v7 (Edgar 2013) as above; and (iii) three alternative quality filtering parameters in Usearch (Maxee $=0.5$; Maxee $=1$; without Maxee). Processed sequences were OTU clustered with three different algorithms: Usearch (greedy heuristic approach; Edgar 2013) (cluster_otus option); CROP (Bayesian approach; Hao et al. 2011); and Swarm (agglomerative approach; (Mahé et al. 2014) (Suppl. Fig. S2).

The combination of 24 read processing settings and 3 OTU clustering methods yielded a total of 72 OTU sets for each of the three metabarcode fragments. For each DNA fragment (bc5', bc3', SSU) we estimated the number of exclusive and shared OTUs between each pair of the 72 OTU sets obtained. This required that we possess a list of all OTUs present in the various sets. We assembled the 72 OTU sets with a minimum similarity threshold of 3\% in Geneious v7.1.9 (Kearse et al. 2012). The resulting assemblies were exported as a 50\% majority rule consensus sequence to represent each OTU, and used as references to subsequently map the OTUs obtained for each of the 72 OTU sets under a $3 \%$ similarity threshold, using the command-usearch_global. The proportion of shared and exclusive OTUs in each pair of the 72 OTU sets were estimated from the OTU table using R.

Based on the results from these tests (below), we used the following parameters for all further analyses: reads were denoised using BFC ( $\mathrm{Li} 2015$ ), and processed following several steps of the Usearch (Edgar 2013) pipeline: reads were merged (option mergepairs-fastq_minovlen 150 (130 for $\left.b c 3^{\prime}\right),-f a s t q \_$maxdiffs 30), quality filtered (Maxee=1), dereplicated (-derep_fulllength) and sorted (sortbysize options). Sequences with only one read (-minsize 2) were excluded, and a de novo chimera checking was conducted (-uchime_denovo option).

\section{De novo OTU generation from metabarcoding}

Quality-filtered metabarcode sequences were subjected to clustering with each of the three clustering algorithms using $3 \%$ and $10 \%$ dissimilarity thresholds for the coxl bc5' and bc 3 ' fragments, and 3\% threshold for the $S S U$ gene fragment. Each OTU set was filtered to retain only OTUs, which corresponded to the targeted invertebrates and to remove bacterial and other sequences. For this

This article is protected by copyright. All rights reserved. 
purpose, Geneious was used in batch mode to align each OTU set (the representative sequence, i.e. the centroid according to Usearch) with MAFFT options FFT-NS-2 (Katoh \& Standley 2013) and to generate an UPGMA tree based on Tamura-Nei distances. Only the OTUs included in the largest clade of the UPGMA tree within a 30\% dissimilarity threshold were retained, and all other sequences presumably not representing the targeted gene fragment were removed with a custom R script using the libraries ape (Paradis et al. 2004), rncl and stringr (Wickham 2013). Finally, the retained coxl sequences were aligned in Geneious using MAFFT and the Translation Align option, and subsequently sequences with insertions, deletions or stop codons disturbing the reading frame were excluded.

\section{Identification of OTUs against NCBI and the BOLD-OTUs reference set}

Assignments of OTUs to high level taxonomic categories were conducted with the lowest common ancestor (LCA) algorithm implemented in Megan v5 (Huson et al. 2007). Each OTU representative sequence was subjected to BLAST searches against the NCBI $n t$ database (December 2016; blastn outfmt 5 -evalue 0.001). BLAST matches were fed into Megan to compute the taxonomic affinity of each OTU. We accepted the taxonomic ranks in the NCBI Taxonomy database (December 2016) and Megan was used to estimate richness for phyla within Metazoa, classes within Arthropoda, and orders within Insecta and Crustacea.

Secondly, the identifications were conducted against BOLD-OTUs. Analyses were based either on metabarcoding reads only, after filtering, or after the de novo OTU clustering step described earlier. Processed reads of $b c 5^{\prime}$ and $b c 3^{\prime}$ were matched against BOLD-OTUs using the -usearch_global option with the same 3\% threshold. The python script uc2otutab.py was used to generate a list of matched OTUs. For the identification of de novo OTUs from clustering with Usearch, Swarm and CROP the same protocol of matching the BOLD-OTUs in the reference database was applied to the representative sequences from each OTU (at 3\% threshold).

\section{Community composition and indicative species analyses}

Ecological statistical analyses for total beta diversity and the associated turnover and nestedness components (Baselga 2010) across sampling sites were conducted for the Metazoa, Arthropoda, Insecta and Crustacea sub-datasets. Community composition tables (OTU x site tables) were obtained by matching Illumina processed reads (blastn-outfmt 5 -evalue 0.001) against (a) the selected sets of de novo OTUs obtained with Usearch (3\% and 10\% similarity thresholds for $b c 5^{\prime}$ and $b c 3^{\prime}$, and $3 \%$ for $S S U$ ); and (b) the BOLD-OTUs reference dataset (for $b c 5^{\prime}$ and $b c 3^{\prime}$ gene fragments, only for

This article is protected by copyright. All rights reserved. 
Arthropoda, Insecta and Crustacea sub-datasets). When using de novo generated OTUs, taxonomic assignments obtained in Megan (see above) were used to extract sub-datasets of OTUs identified as (a) Metazoa, (b) Arthropoda, (c) Insecta and (d) Crustacea with an LCA parameter value of 90. Likewise, the BOLD-OTUs reference sequences were taxonomically assigned to Arthropoda, Insecta and Crustacea sub-datasets.

Taxonomic subsets of data were used to conduct analyses of community composition, either for the meio- and macro-fauna samples separately or after combining both samples from the same site and time as a single sample. Distance matrices by pairs of sites were generated for total beta diversity (Sorensen index), turnover (Simpson index; species replacement, without the effect of variation in richness) and nestedness (Sorensen - Simpson index; pure richness effect) using the R library betapart (Baselga \& Orme 2012), and the R library vegan (Oksanen et al. 2013) was used to perform Nonparametric Multidimensional Scaling analyses (NMDS). Analyses were plotted with the ordisurf option to generate clines based on richness values and ordispider to connect the samples from upstream and downstream of the spill. Finally, a Permutational Anova (Permanova) was conducted using the function adonis, and the significance of differences was assessed using a stress test and the envfit test of correlation. Jaccard distances were used to verify the existence of significant differences in the communities upstream and downstream of the spill. We additionally used the mvabund package for the analysis of multivariate abundance data (Wang et al. 2012) to test upstream and downstream effects (factor spill) on community composition (manyglm function) and to identify which individual species show significant differences in their distribution between control and impacted sites (anova.manyglm function; test=LR, $\mathrm{nBoot}=999$ ) using the results from the reference database (taxonomy-dependent) approach. Finally, we used Dufrene-Legendre indicator species analysis (indval) (Dufrêne \& Legendre 1997) using the R package labdsv (Roberts 2007) to compare control versus impacted sites.

\section{RESULTS}

\section{Sequencing, read processing and OTU clustering}

OTU delimitation was conducted on tens of thousands of Illumina (2x300 bp) sequence reads per sample from both the meio- and macro-fauna extracts (ranging 41,306 - 177,653 for $b c 3$ '; 5,487 100,344 for $b c 5$ '; and 34,555 - 107,016 for SSU) (Suppl. Table S1). A single sample (Impacted site 1Time 1: IS1-T1) failed to produce a PCR product and was removed from the analysis. For the remaining 11 samples, Spearman's rho correlation tests revealed no correlation between raw read numbers and the number of OTUs classified as Metazoa for the three DNA fragments (Suppl. Fig. S3), i.e. different read abundances between samples did not affect the number of OTUs recovered.

This article is protected by copyright. All rights reserved. 
The raw reads were subjected to basic quality filtering, which reduced the usable reads to about $90 \%$ of the initial reads for the $S S U$ marker, while for both coxl markers in many samples a much greater proportion was lost, sometimes retaining only $10-20 \%$ of reads. There were no systematic differences in total read numbers after quality filtering between upstream and downstream or between meio- and macro-fauna samples (Suppl. Table S1). The paired-end merging of R1 and R2 (forward-reverse) reads resulted in a further reduction, but was broadly uniform across samples (Suppl. Table S1). Various modifications of the basic protocol for quality filtering and read merging under 24 different parameter settings were applied. The resulting merged reads were then subjected to OTU clustering using three different clustering methods (Suppl. Fig. S2). After excluding OTUs not matching the expected gene fragments or taxonomic groups using a tree-based method (see Materials and Methods), the number of OTUs under these various parameter settings ranged from 479 to 810 for the $b c 5^{\prime}$ fragment, from 543 to 1150 for $b c 3^{\prime}$, and from 193 to 590 for SSU (Suppl. Table S2).

The proportion of shared and exclusive OTUs obtained (for each gene) showed broadly similar results across the parameter settings (Fig. 2). The OTUs obtained with different clustering methods (Usearch, CROP and Swarm) were shared in $91.1 \%$ and $92.7 \%$ of the cases for the $b c 5^{\prime}$ and $b c 3^{\prime}$ fragments, respectively, with the major difference attributed to the CROP clustering method that lacked $4.6 \%$ of OTUs obtained with the other methods. The same trend was observed for $S S U$, where a $65.6 \%$ of the OTUs were generated with the three methods and an additional $20.3 \%$ were shared between Usearch and Swarm, but not with CROP. The implementation of a denoising tool (BFC software) resulted in a moderate effect on the number of obtained OTUs ( $~ 90 \%$ of OTUs shared), except for $s=20$ which resulted in the absence of $30 \%-50 \%$ of the OTUs. The use of the Maxee filtering option also produced a moderate effect on OTU recovery ( $90 \%$ of OTUs shared among parameters settings). Finally, using PEAR or Usearch for the merging step resulted in $\sim 90 \%$ shared OTUs (Fig. 2).

\section{Taxonomic profiles based on de novo OTUs using Megan}

Next, de novo OTUs were identified against the Genbank database via LCA assignment to major taxon (see Materials and Methods). We only assessed the OTU set obtained under a single representative parameter setting (BFC with $s=0.5$ for read denoising, Maxee $=1$ for read filtering, and mergepairs in Usearch for read merging), to which we applied the three clustering methods (Usearch, Swarm, CROP) under similarity thresholds of $3 \%$ and $10 \%$ for the coxl fragments, and $3 \%$ for the $S S U$ fragment (Fig. 3). In the $b c 5^{\prime}$ and $b c 3^{\prime}$ fragments, the LCA assignment showed that samples were dominated by OTUs of Arthropoda, followed by Rotifera and Annelida. Within Arthropoda, Insecta was the most abundant group, followed by Arachnida and various classes within the subphylum Crustacea (Fig. 3). The Insecta were dominated by Diptera, followed by Coleoptera, Ephemeroptera and Trichoptera. In the SSU data, the OTU composition was also dominated by Arthropoda but the

This article is protected by copyright. All rights reserved. 
total number of OTUs was substantially lower compared to those from coxl, in particular in the Insecta and specifically the Diptera, which was reduced to approximately $1 / 4^{\text {th }}$ of the $b c 3^{\prime}$, and $b c 5^{\text {' }}$ OTUs (Fig. 4; Suppl. Table S3). In Crustacea, both genes detected the major orders such as Podocopida, Isopoda, Amphipoda, Cyclopoida and Diplostraca. OTU numbers were reduced in SSU compared to the coxl marker in Annelida, and Rotifera were not detected with $S S U$ at all, despite the recovery of several dozen OTUs with coxl. In contrast, the SSU dataset retrieved a similar number of OTUs of Mollusca, while for Platyhelminthes and Nematoda most OTUs were only detectable with the SSU marker (Suppl. Table S3). Taxonomic profiles obtained at a 10\% similarity threshold showed the same trend as described above, but with a substantial reduction in the total number of OTUs (Fig. 4; Suppl. Table S3).

\section{Taxonomic assignment against the $B O L D-O T U$ reference database}

Direct mapping of sequence reads against the reference set resulted in matches to 207 BOLD-OTUs. The majority of these BOLD-OTUs matched a unique BIN in the BOLD database (Table 1 for Diptera and Suppl. Table S4 for the complete dataset). Eighteen of these 207 BOLD-OTUs included a single sequence, but were not included by BOLD in their BIN system. In two cases, a pair of BOLD-OTUs produced separate clusters of sequences from a single BIN, and data for these were lumped, to maximise consistence with BOLD. Read numbers for each OTU varied by four orders of magnitude, to a maximum of $>20000$ reads for some species of Baetidae. Out of a total of 207 BOLD-OTUs with read matches, 36 OTUs were obtained exclusively with the $b c 5^{\prime}$ fragment and 46 OTUs with the $b c 3^{\prime}$ fragment, but in most of these cases only a few reads $(<10)$ were obtained.

Next, we mapped the de novo generated OTUs against the BOLD-OTUs. Species detections obtained in this way closely matched those from the read mapping, although the method was slightly less sensitive in cases of low number of reads that did not produce an OTU (Table 1, Suppl. Table 4). Species detection depending on the three clustering methods (Usearch, Swarm, CROP) or marker $\left(b c 5^{\prime}\right.$ and $\left.b c 3^{\prime}\right)$ was generally consistent, and observed differences tend to occur for species with low read counts (Fig. 4; Suppl. Table S4).

The total numbers of identified OTUs from read matching and de novo clustering were generally similar, in particular in Diptera for both $b c 5^{\prime}$ and $b c 3^{\prime}$. In other taxonomic groups, the OTU counts from read matching were closer to those at the $10 \%$ thresholds of the de novo OTU analysis or even lower, as in the case of several classes and orders within Crustacea (Fig. 4; Suppl. Table S3), whose

This article is protected by copyright. All rights reserved. 
reference databases were less complete. The reduction at the $10 \%$ threshold generally affected cases that were split into multiple OTUs at 3\%; for some of these cases, the sequence reads also matched several BOLD-OTUs but based on taxonomic assignments were identified as the same species, e.g. some of the eight BOLD-OTUs identified as the isopod Asellus aquaticus (Fig. 4; Suppl. Table S4).

Comparisons with the taxon list of macroinvertebrates provided by traditional analysis (Thompson $e t$ al. 2016) were made for Arthropoda based on identifications by read mapping with the $b c 5^{\prime}$ and $b c 3^{\prime}$ (coxl) fragments at the $3 \%$ threshold level. The identifications included many exact matches to the Thompson et al. (2016) list, although the latter included only 38 arthropod taxa in total, of which 11 taxa were not represented in the OTUs dataset (not even at higher taxonomic level). The missing taxa were from a range of higher taxa, including Ephemeroptera, Plecoptera, Coleoptera, Isopoda, Arachnida and others, while other species in these taxa were easily recovered by the coxl metabarcodes. In many cases, Thompson et al. (2016) did not separate the entities at the species level. Notably the Chironomidae, which were listed as two taxa (Chironomidae and subfamily Tanypodinae) in Thompson et al. (2016) were split into a total of 55 OTUs in the current analysis and most could be identified to species. Similarly, the single entry for Limnephilidae in Thompson et al. (2016) was split into 5 OTUs, the entry for Dytiscidae into 3 OTUs, and even genus-level entries were split further, e.g. Baetis sp. corresponded to 9 OTUs and Simulium sp. corresponded to 4 OTUs in the coxI analysis. In other cases, several BOLD OTUs were assigned the same binomial (although not unequivocally in all cases; see Suppl. Table 4), indicating the high intraspecific diversity on some species or even the possible existence of cryptic species.

\section{Effect of the spill on community composition and indicative species}

The presence or absence of species in various metabarcoding samples was used to establish the turnover and nestedness of assemblages above and below the spill site. NMDS analyses for total beta diversity, nestedness and turnover across sampling sites were conducted for the Metazoa, Arthropoda, Insecta and Crustacea datasets for: (a) OTUs at 3\% and $10 \%$ for $b c 5^{\prime}$ and $b c 3^{\prime}$; (b) OTUs at $3 \%$ for $S S U$; and (c) for the BOLD reference dataset and $b c 5^{\prime}$ and $b c 3^{\prime}$ gene fragments (summarized in Table 2). Significant differences in community composition were detected in all cases between impacted and control sites that were mainly driven by a high turnover of species, but not for the nestedness component of beta diversity (Fig. 5, S4-S9). Results were very similar for de novo clustering with BLAST+Megan for OTU classification and reads based approaches using BOLD-OTUs for identification (Fig. 6, Table 2). The NMDS plots conducted on the meio- and macro-faunal fractions separately also showed very similar patterns (Fig. 7).

This article is protected by copyright. All rights reserved. 
Of the 207 OTUs detected by matching the reads to BOLD-OTUs, 170 were obtained from the meiofauna sample, against only 126 OTUs from the macrofauna fraction (filtration at $>1 \mathrm{~mm}$ ), and 70 OTUs were unique to the meiofauna. There were clear patterns in the distribution of meio- and macrofaunal samples, for example showing that Brachiopoda, Collembola and some Arachnida were almost entirely found in the former, reflecting their small body size, but most large-bodied insects were also recovered in the meiofauna fraction (in addition to the macrofaunal fraction). Second, combining the total species count of meio- and macro-faunal fraction, the control sites had slightly more species compared to the impacted sites but both had a high proportion of unique OTUs (65 versus 53)

Indval analyses for strong effects of the spill revealed a statistically significant indicative value of Gammarus pulex associated to control sites (indval $\mathrm{p}<0.05$ ), while Asellus aquaticus and several Chironomidae species (Tanytarsus eminulus, T. brundini, T. pallidicornis, T. ejuncidus, Cricotopus bicinctus, Paratendipes albimanus) were associated to impacted sites (indval p<0.05) (Table 3). Mvabund analyses resulted in significant differences for Asellus aquaticus and Tanytarsus eminulus $(\mathrm{p}<0.05)$. Additional tests conducted with the indval function to identify indicative species associated to the two sampling periods resulted in the Ephemeroptera Centroptilum luteolum as indicative of sampling period 2 (2.5 months after the spill), whereas when the factor spill and the sampling period were considered in combination, two Chironomidae (Eukiefferiella claripennis and Cricotopus bicinctus) and one Branchiopoda (Chydorus sphaericus) were found as indicative of the impacted sites at the sampling period 2 .

\section{DISCUSSION}

Metabarcoding revealed high species turnover between control and pesticide-impacted sites in the River Kennet, in line with the earlier study based on conventional taxonomic assignments (Thompson et al. 2016), but with far more complete and taxonomically resolved data. This was also achieved at a fraction of the cost and time, although absolute abundance data were sacrificed as a result (note that most routine biomonitoring approaches only ever use relative abundance anyway; Friberg et al. 2011; Gray et al. 2016). The high read depth and accuracy of Illumina sequences, in addition to an increased completeness of reference databases, now can determine the identity of taxa as effectively as conventional biomonitoring, but with the added advantage of capturing data at the species level for virtually all components of the sample - including both the temporary and permanent meiofauna, which are very diverse and abundant yet are routinely ignored due to difficulties in their identification via traditional microscopy (Friberg et al. 2011; Gray et al. 2016). Our study takes a further step towards this explicit species level approach, by addressing two key issues: the selection of appropriate gene regions and universal primers for metabarcoding, and the choice of the most appropriate bioinformatics tools and parameter settings. If linked with existing DNA databases, metabarcoding

This article is protected by copyright. All rights reserved. 
provides the species level resolution of community composition, to identify the indicators of disturbed and undisturbed sites and, ultimately, to bridge biomonitoring of community structure and ecosystem functioning.

\section{Methodological decisions: choice of gene markers and bioinformatics}

Researchers are confronted with a wide range of options for data processing and parameter settings at all stages of metabarcoding. The final outcome is affected by the early steps of the wet lab procedures for DNA extraction from various environmental samples and the choice of PCR primers. The two genetic markers used here amplifying portions of the mitochondrial coxl and the nuclear 18S rRNA genes illustrate the important effects of marker choice, affecting: amplification of major taxonomic groups, power of separation of species, and the completeness of available reference data. Based on these criteria, the barcode fragment of the coxl gene remains the most powerful choice for metabarcoding studies of animal communities. The advantage of using the coxl gene is due to (i) the benefits from an already available and growing database for this marker from standard barcoding; (ii) the widely accepted standardization of the marker choice, allowing for a comparison of different studies; and (iii) the demonstration that universal primers recover complex communities composed of a taxonomically wide range of arthropods and other metazoans.

The primers used here for both coxl fragments produced rather similar results for total OTU numbers and taxonomic distribution of major groups, indicating that amplification from mixtures is largely reliable. Differences between OTU detection with both primers are mainly at the level of individual species, which requires further investigation, but possibly is due to variation among individual PCR on low-abundance (low biomass) species, as reflected in the fact that disagreements are mostly due to OTUs represented by low numbers of reads. In contrast, the $S S U$ (rRNA) marker is far less variable than mitochondrial DNA and thus presumably closely related species are collapsed into single OTUs (Tang et al. 2012). This was evident from the lower number of OTUs obtained, in particular for groups containing multiple congeneric species, such as the Chironomidae. However, in addition to the lumping of close relatives, the overall taxonomic profiles also differed strongly, presumably due to the greater conservation of $S S U$ primer binding sites across phyla. Specifically, Platyhelminthes and Nematoda were only recovered with $S S U$, whereas the efficiency was lower for Arthropoda. The former groups are rarely if ever considered in traditional biomonitoring, whereas arthropods underpin most schemes around the world. These well-known issues of taxonomic resolution and differences in the spectrum of PCR amplification breadth need to be considered carefully depending on the target taxa. It is unlikely that truly universal primers can be found for amplification of all animal phyla or that a single locus can separate species universally. Even so, poor amplification of some groups for coxl possibly can be alleviated with different primer sequences, at least for regions that are partly

This article is protected by copyright. All rights reserved. 
overlapping, using multiple PCRs on the DNA extracts, to be included in the Illumina libraries with relatively little added effort. An alternative is the use of slightly more conserved markers, such as the mitochondrial rRNA (12S and 16S) genes suggested in recent metabarcoding studies, which present a compromise between broad, unbiased PCR and species-level differentiation (Taberlet et al. 2012), but this approach misses the advantage of comparisons against the available sequence databases for the barcode coxl gene fragment.

For the bioinformatics processing, different parameter settings had little effect. Different clustering algorithms employing fundamentally different methodology generated largely uniform OTUs, except perhaps the CROP algorithm, which detected slightly fewer OTUs than the Usearch and Swarm methods. Equally, the first step of denoising was robust over a range of parameters that are broadly within the boundaries of most published applications of the software. The read pairing step and the final filtering based on the error frequency in the reads (the maximal expected error, Maxee) only changed the outcome of the final assembly for $<10 \%$ of OTUs. Presumably, the denoising procedure mainly eliminates minor variants among the reads that are subsumed into the OTUs at the minimum clustering threshold of $3 \%$ that was applied here, and thus their prior elimination has little impact on the final outcome of the OTU delimitation. We also find that many OTUs are generated that are either non-mitochondrial or correspond to non-target taxa, including bacteria. These were removed via a phylogenetic approach using rapid UPGMA trees and retaining only the major target clade for metazoans, thus avoiding later complications with species counts and turnover analysis. As a general rule, stringent parameters settings should be applied, but the appropriate parameter space is fairly wide and so its reassessment is not necessary for each study; for comparability, a particular parameter set should be chosen and applied consistently.

\section{Species definitions in de novo clustering and read mapping}

A key aspect of the metabarcoding procedure is the recognition and identification of the species in the sample, which requires a valid taxon concept for each species based on solid data for species circumscription (Wheeler 2004). The clustering algorithms define the species limits based on similarity thresholds, akin to the search for a barcoding gap in standard (Sanger) barcoding (Meyer \& Paulay 2005), but the actual variation is overlain by sequencing read errors, and true variants from variable mitochondrial copies (heteroplasmy) and nuclear mitochondrial insertions (numts). While the various clustering algorithms broadly agreed on the number and extent of OTU delineations, differences in particular species hypotheses were evident and dependent on the threshold. If set to $3 \%$ in the coxl marker, OTU delineation largely reflected the species numbers expected from the Linnaean taxonomy, whereas the highly conservative $10 \%$ threshold lumped many species. The application of more refined algorithms to the OTU circumscriptions is desirable, although coalescence

This article is protected by copyright. All rights reserved. 
based procedures including GMYC and PTP (Pons et al. 2006; Zhang et al. 2013) currently are not easily applicable to the very large number of reads. In addition, metabarcoding sequences are comparatively short, which limits the resolving power of these sequences in any species delimitation procedure.

The two principal approaches used here for generating community OTU lists, i.e. the 'taxonomy independent' approach based on de novo generated OTUs, and the 'taxonomy dependent' approach via read mapping against the reference database (Schloss \& Westcott 2011) produced generally similar results (although with slightly fewer entities detected in the latter). When working with de novo generated OTUs, special attention should be paid to the method used for classifying OTUs. Megan has been proven as a useful tool based on Blast searches against reference databases, as here conducted. Nevertheless, incompleteness of reference databases can result on inaccurate classifications when relaxed similarity thresholds are considered (e.g. 70\%), whereas higher similarity stringency (about 90\%) will result in many OTUs with no taxonomic assignation. This is here illustrated by the classification of one OTU retrieved independently with both coxl fragments as Echinodermata, a marine group not expected to be present in the target sample. This identification corresponds to a few matches with similarity below $80 \%$ and the sequence requires several indels to align to the identified reference. For sequences with such a weak match, Megan may use similarly weak matches with several other reference sequences from distantly related taxa, to return an identification at the deepest taxononomic level corresponding to all of these weak matches. If the focal sequence has no close match in the database, spurious Blast similarities might indicate a best match in a certain area of the reference database, despite representing a lineage quite divergent from these best matches, and thus this sequence is not necessarily related to Echinodermata.

In the read mapping approach, the problem of species delimitation is shifted to the reference databases that define intraspecific variation and link a set of sequences to a Linnaean binomial. In the European freshwater arthropods we could heavily draw on the relatively good species representation in BOLD. For simplicity, we downloaded all available sequences and conducted OTU clustering on these sequences, in analogy to the metabarcode data, to produce a reference database (the BOLD-OTUs), after which only those with similarity to the metabarcoding sequences encountered in the target samples were considered further (a total of 207 OTUs). The BIN groups presented in the BOLD database are an alternative compilation of the existing reference data, and we established that they produce similar OTU circumscriptions as those from the Usearch clustering at a $3 \%$ similarity threshold. Both compilations show a good general congruence with the Linnaean names, but in several cases multiple BINs and BOLD-OTUs were associated to a single binomial, probably reflecting high intraspecific variation in some species whose real species limits need to be evaluated with additional information. The relevance of these closely related OTUs remains unclear but may be related to the presence of multiple divergent mitogenome copies in the focal species. For example,

This article is protected by copyright. All rights reserved. 
some isopods, including A. aquaticus, exhibit atypical mitogenomes composed of duplicated regions that apparently are maintained constitutively as heteroplasmic copies (Doublet et al. 2012). Other cases, such as the ephemeropteran Baetis rhodani or the amphipod Gammarus pulex, are already well established species complexes consisting of multiple cryptic species (Karaman \& Pinkster 1977; Williams et al. 2006; Rutschmann et al. 2014; Bisconti et al. 2016). Taxonomic difficulties associated to these cases may affect the reference sequence databases, such the detection of the oriental Gammarus nekkensis with the $b c 5^{\prime}$ fragment, whereas G. pulex is exclusively found with $b c 3^{\prime}$. It should be noted that G. pulex is represented by 5 different BINs at BOLD, whereas G. nekkensis forms up to 10 BINs (accessed 13-06-2017). This taxonomic and molecular complexity highlights the need of a careful assessment by expert taxonomists and molecular biologist for consensus species delineation. Yet, beyond these taxonomically complicated cases, the existing barcode database already holds an excellent coverage for European aquatic macroinvertebrates.

Whereas the direct read mapping approach could circumvent the problematic step of OTU clustering, maximising comparability between different studies, OTUs from the de novo clustering could still be useful in particular to identify species not yet embedded in reference databases, albeit without link to a Linnaean name. The robustness of the de novo approach shown here therefore offers a defensible option for species assemblages whose coverage of reference sets is still patchy, and could be used in biodiversity discovery in hitherto unstudied ecosystems. Ideally, standardized protocols should use a combined approach, where direct mapping for biomonitoring is complemented with de novo OTU clustering under defined parameters and a comparison of OTUs with reference databases. This can help to identify gaps in the reference database, through an iterative process, contributing to refine local reference databases. Once a validated reference set is in hand, the straightforward mapping of sequence reads against these DNA-based grouping provides easily repeatable and stable species identification for biomonitoring. This provides a reliable link to taxonomy and resolution to species level even in the most problematic taxa, such as Chironomidae, which can be used to improve conclusions reached by river monitoring programs, and subsequently to improve conservation and management practices (e.g., Chironomidae richness can be stable while high turnover happens after an impact). In future, through the direct link with taxonomic identifications, metabarcoding can then be connected to traits databases available for a large proportion of aquatic invertebrates species in Europe (Tachet et al. 2002; Schmidt-Kloiber \& Hering 2015), to bridge the critical gap that still exists between structural biodiversity and functional measures related to ecosystem processes (Friberg et al. 2011; Woodward et al. 2013).

This article is protected by copyright. All rights reserved. 


\section{Implications for biomonitoring and ecological studies}

The total diversity of identified species in our metabarcoding study far exceeds the number of invertebrate species detected using conventional analyses (Thompson et al. 2016) - by at least a factor of 5 - even under the most conservative OTU detection parameters. Three aspects contribute to the high numbers: (i) the greater taxonomic resolution compared to the morphological analysis that was conducted on higher taxonomic levels, especially for the Chironomidae; (ii) the split of binomial species names into multiple molecular OTUs (at the 3\% threshold in cox1); and (iii) the better detection of minute specimens, especially those in the temporary meiofauna, in part representing early instars of otherwise larger-bodied species, which are often overlooked in visual assessments, even under light microscopy. These factors thus resolve some of the drawbacks of conventional techniques, namely the incomplete species identification, poor separation of cryptic diversity, and incomplete sampling of the freshwater assemblage. The study also highlights some of the remaining challenges of generating complete metabarcoding inventories: the problem of lumping and splitting of Linnaean species, the low primer efficiency for particular taxa, the variation among PCR runs, and the sampling itself which is affected by stochastic error. Our sample consisted of two independent Surber samples each, from three sites above and three below the impact zone. The methodology is now sufficiently well developed to be applied to many more samples, and denser sampling may reveal additional species that show a clear response to environmental conditions. Ultimately these methods should be applicable in a highly consistent manner for regulatory purposes, perhaps after matching these data against existing schemes and indexes for assessing water quality. For instance, the $600+$ pre-defined reference stream sites used for the UK-wide RIVPACS biomonitoring scheme would offer an ideal testbed for this 'next-generation biomonitoring' approach (Bohan et al. 2017), as would the UK Acid Waters Monitoring Network species-level data that now span several decades and multiple standing and running waters (e.g. Gray et al. 2016).

Our analyses confirmed the previously observed shifts in community composition, despite the variation among individual samples, as the ordinations clearly separated samples from the control and impacted sites. This can be discerned at various taxonomic levels, for different arthropod classes, for meio- and macro-fauna, and for the communities established with each of the three markers. The detailed species list now complements this broad-scale information with the traditional approaches, and in particular it shows that the most resilient $r$-selected taxa, such as chironomids, recover most rapidly to occupy vacant niches following the crashes in $K$-selected taxa. Our study confirms that this increase is indeed an increase in species richness, not just in abundance of species present already at lower density. The conventional approach, as is common in freshwater ecology, simply lumped these highly responsive and speciose taxa into a single entity that revealed marked increases in abundance in the absence of potential competitors and predators, but provided no information on chironomid biodiversity. Most of the taxa in the impact samples are orthoclads (grazers on stones and plant

This article is protected by copyright. All rights reserved. 
surfaces), with a few others that are detritivores living in soft sediments, in addition to some predatory species. The increase of several species of Tanytarsus, a group of dominant sediment-dwelling detritus feeders that have been shown to feed on diatoms in the early larval stages (Ingvason et al. 2004), is consistent with the increase of several large diatom species observed in the post-spill sites (Thompson et al. 2016). These preliminary data suggest that inferences about the ecological response in terms of both impacts and resilience is masked by limited taxonomic resolution, and that this in turn is likely to reflect marked functional trait shifts that are overlooked in routine biomonitoring schemes. Biomonitoring at present is focused on responses to organic pollution or, to a lesser extent, acidification, whereas responses to other stressors are still poorly characterised in natural systems: the next-generation biomonitoring approach we use here could open the door to improving the sensitivity and power of detection in relation to both response variables and a wider range of drivers than is currently possible..

\section{Conclusions}

Our study refines the parameter space of metabarcoding studies generally, and our specific case study highlights its potential for next-generation biomonitoring to advance the current state-of-the-art assessment of water quality and ecological status. At least in terms of detecting relevant changes in community, neither the marker, the read processing or the clustering method or threshold affected our ability to detect the spill's impact on the community. The availability of full species-level inventories for the first time enabled us to exploit the extensive ecological databases that are now available for freshwater species in Europe, and also to begin to elucidate relevant trait differences. In addition, the capacity to use all taxa, rather than a narrow subset for which taxonomic expertise is available, promises to deliver a far more informative and mechanistic understanding of biodiversity in freshwater ecosystems and its responses to environmental stressors.

\section{Acknowledgements}

This study was funded by NERC grant NE/M021955 to DYW, APV and KB. We are grateful to Steve Brooks, Benjamin Price and Tjorborn Ekrem for discussions about species responses in freshwater communities.

\section{DATA ACCESSIBILITY}

Raw metabarcode data deposited in Dryad: doi:10.5061/dryad.104kg

This article is protected by copyright. All rights reserved. 


\section{AUTHORS'S CONTRIBUTIONS}

APV and GW conceived the study; CG and GW collected the samples; CA, PA, DWY and APV designed the analyses; CA and PA did the molecular lab work and performed the analyses; CA and APV led the writing and all author contributed to the discussion of the results and the final writing of the manuscript

\section{SUPPLEMENTARY MATERIALS}

\section{Information on PCR reagents and conditions}

\section{Supplementary figures}

\section{Supplementary tables}

3. Code for metabarcoding pipelines, read processing and clustering

Table 1. BINs of Diptera from BOLD identified based on usearch_global searches under a 3\% similarity threshold of (i) processed reads and (ii) OTUs clustered at 3\% (details in text).

\begin{tabular}{|c|c|c|c|c|c|c|c|c|c|c|c|c|}
\hline BIN & Family & Main species id of BIN & bc-5' & bc-3' & $r-b c-51 r$ & r-bc-3' & C & 1 & MC & MS & T1 & T2 \\
\hline BOLD:AAJ7051 & Agromyzidae & Agromyza pseudoreptans [19] & $-/-/-/-$ & $\mathrm{R} / \mathrm{U} / \mathrm{C} / \mathrm{S}$ & 0 & 5 & & b3 & b3 & & b3 & \\
\hline BOLD:ACI4790 & Bibionidae & Dilophus febrilis [21] & $R / U / C / S$ & $R / U / C / S$ & 478 & 428 & b5 b3 & & b5 b3 & b5 & b5 & b5 b3 \\
\hline BOLD:ACP0608 & Cecidomyiidae & Cecidomyiidae [3] & $\mathrm{R} / \mathrm{U} / \mathrm{C} / \mathrm{S}$ & $\mathrm{R} /-/-/ \mathrm{S}$ & 455 & 31 & & b5 b3 & & b5 b3 & & b5 b3 \\
\hline BOLD:ACS1169 & Ceratopogonidae & Palpomyia flavipes [6] & R/U/-/- & $\mathrm{R} / \mathrm{U} / \mathrm{C} / \mathrm{S}$ & 20 & 41 & b5 b3 & b5 b3 & 3 b5 b3 & b5 b3 & b5 b3 & 3 b5 b3 \\
\hline BOLD:ACD1957 & Chironomidae & \multicolumn{2}{|c|}{ Apsectrotanypus trifascipennis [9]R/U/C/S } & $\mathrm{R} / \mathrm{U} / \mathrm{C} / \mathrm{S}$ & 81 & 1446 & b5 b3 & b5 b3 & 3 b5 b3 & b5 b3 & & b5 b3 \\
\hline BOLD:ADE2432 & Chironomidae & Brillia bifida [6] & R/-/-/- & $-/-/-/-$ & 1 & 0 & & b5 & & & b5 & \\
\hline BOLD:ACR1089 & Chironomidae & Chironomidae & $R / U / C / S$ & $\mathrm{R} / \mathrm{U} / \mathrm{C} / \mathrm{S}$ & 550 & 93 & b5 b3 & b5 b3 & 3 b5 & b5 b3 & b5 b3 & 3 b5 b3 \\
\hline BOLD:ACP8764 & Chironomidae & Chironomidae [13] & $\mathrm{R} / \mathrm{U} / \mathrm{C} / \mathrm{S}$ & $-/-/-/-$ & 130 & 0 & & b5 & b5 & b5 & & b5 \\
\hline BOLD:ACP6740 & Chironomidae & Chironomidae [60] & $\mathrm{R} /-/-/-$ & $\mathrm{R} / \mathrm{U} / \mathrm{C} / \mathrm{S}$ & 11 & 9 & & b5 b3 & 3 b3 & b5 b3 & & b5 b3 \\
\hline BOLD:ACP2182 & Chironomidae & Chironomidae [92] & $\mathrm{R} / \mathrm{U} / \mathrm{C} / \mathrm{S}$ & $\mathrm{R} / \mathrm{U} / \mathrm{C} / \mathrm{S}$ & 33 & 26 & & b5 b3 & & b5 b3 & & b5 b3 \\
\hline BOLD:AAW5799 & Chironomidae & Conchapelopia hittmairorum [3] & R/-/-/- & R/-/-/- & 1 & 4 & & b5 b3 & & b5 b3 & & b5 b3 \\
\hline BOLD:AAP5886 & Chironomidae & Conchapelopia melanops [11] & $R / U / C / S$ & $R / U / C / S$ & 1018 & 331 & b5 b3 & b5 b3 & 3 b5 b3 & b5 b3 & b5 b3 & 3 b5 b3 \\
\hline BOLD:ACQ3496 & Chironomidae & Conchapelopia pallidula [1] & $\mathrm{R} / \mathrm{U} / \mathrm{C} / \mathrm{S}$ & $-/-/-/-$ & 3 & 0 & & b5 & & b5 & & b5 \\
\hline BOLD:ACD1670 & Chironomidae & Corynoneura sp. & $\mathrm{R} / \mathrm{U} / \mathrm{C} / \mathrm{S}$ & $\mathrm{R} / \mathrm{U} / \mathrm{C} / \mathrm{S}$ & 1178 & 1263 & b5 b3 & b5 b3 & 3 b5 b3 & b5 b3 & & b5 b3 \\
\hline BOLD:ACT8698 & Chironomidae & Corynoneura sp. [6] & $\mathrm{R} / \mathrm{U} / \mathrm{C} / \mathrm{S}$ & $\mathrm{R} / \mathrm{U} / \mathrm{C} / \mathrm{S}$ & 21 & 72 & b5 b3 & b5 b3 & & b5 b3 & & b5 b3 \\
\hline BOLD:AAW5785 & Chironomidae & Cricotopus albiforceps [139] & $\mathrm{R} /-/-/-$ & $\mathrm{R} /-/-/-$ & 2 & 4 & b5 b3 & & & b5 b3 & & b5 b3 \\
\hline BOLD:AAF2345 & Chironomidae & Cricotopus annulator [19] & $R / U / C / S$ & $R / U / C / S$ & 30 & 14 & & b5 b3 & 3 b5 b3 & b5 & & b5 b3 \\
\hline BOLD:AAP5931 & Chironomidae & Cricotopus bicinctus [1] & $\mathrm{R} /-/-/-$ & $-/-/-1-$ & 60 & 0 & & b5 & b5 & b5 & & b5 \\
\hline BOLD:ACU8677 & Chironomidae & Cricotopus bicinctus [1] & $\mathrm{R} /-/-/-$ & $-/-/-/-$ & 5 & 0 & & b5 & b5 & b5 & & b5 \\
\hline BOLD:AAI6018 & Chironomidae & Cricotopus bicinctus [123] & $\mathrm{R} / \mathrm{U} / \mathrm{C} /-$ & $\mathrm{R} /-/ \mathrm{C} / \mathrm{S}$ & 5751 & 4759 & b5 & b5 b3 & 3 b5 b3* & *b5 b3 & b5 & b5 b3 \\
\hline BOLD:AAT9677 & Chironomidae & Cricotopus bicinctus [27] & $\mathrm{R} / \mathrm{U} /-/ \mathrm{S}$ & $\mathrm{R} / \mathrm{U} / \mathrm{C} /-$ & 123 & 1991 & b3 & b5 b3 & 3 b5 b3 & b5 b3 & & b5 b3 \\
\hline BOLD:AAM5377 & Chironomidae & Cricotopus rufiventris [289] & $R / U / C / S$ & $R / U / C / S$ & 8 & 44 & & b5 b3 & 3 b5 b3 & b3 & & b5 b3 \\
\hline BOLD:AAA5299 & Chironomidae & Cricotopus sylvestris [64] & $R / U / C / S$ & $R / U / C / S$ & 4 & 88 & & b5 b3 & 3 b5 b3 & & & b5 b3 \\
\hline BOLD:AAU2576 & Chironomidae & Cricotopus trifascia [5] & $\mathrm{R} / \mathrm{U} / \mathrm{C} / \mathrm{S}$ & $\mathrm{R} / \mathrm{U} / \mathrm{C} / \mathrm{S}$ & 119 & 126 & & b5 b3 & 3 b5 b3 & b5 b3 & & b5 b3 \\
\hline BOLD:AAE4568 & Chironomidae & Eukiefferiella claripennis [185] & $\mathrm{R} / \mathrm{U} / \mathrm{C} /-$ & $\mathrm{R} / \mathrm{U} / \mathrm{C} /-$ & 164 & 116 & & b5 b3 & 3 b5 b3 & b5 b3 & & b5 b3 \\
\hline BOLD:ACT0982 & Chironomidae & Heterotrissocladius sp. 2sW [2] & $\mathrm{R} / \mathrm{U} / \mathrm{C} / \mathrm{S}$ & $\mathrm{R} / \mathrm{U} / \mathrm{C} / \mathrm{S}$ & 48 & 234 & b5 b3 & b5 b3 & 3 b5 b3 & b5 b3 & b5 b3 & 3 b5 b3 \\
\hline BOLD:AAX3566 & Chironomidae & Macropelopia nebulosa [13] & $\mathrm{R} / \mathrm{U} / \mathrm{C} / \mathrm{S}$ & $\mathrm{R} / \mathrm{U} / \mathrm{C} / \mathrm{S}$ & 29 & 58 & b5 b3 & b5 & b5 b3 & b5 b3 & b5 b3 & 3 b3 \\
\hline BOLD:AAB8862 & Chironomidae & Metriocnemus eurynotus [18] & $\mathrm{R} /-/-/-$ & $\mathrm{R} / \mathrm{U} / \mathrm{C} / \mathrm{S}$ & 3 & 18 & & b5 b3 & & b5 b3 & b5 b3 & \\
\hline BOLD:AAD4167 & Chironomidae & Micropsectra atrofasciata [26] & $\mathrm{R} / \mathrm{U} / \mathrm{C} / \mathrm{S}$ & $\mathrm{R} / \mathrm{U} / \mathrm{C} / \mathrm{S}$ & 826 & 596 & & b5 b3 & 3 b5 b3 & b5 b3 & & b5 b3 \\
\hline BOLD:AAC7823 & Chironomidae & Micropsectra contracta [14] & $\mathrm{R} / \mathrm{U} / \mathrm{C} / \mathrm{S}$ & $\mathrm{R} / \mathrm{U} / \mathrm{C} / \mathrm{S}$ & 62 & 98 & & b5 b3 & 3 b5 b3 & b5 b3 & & b5 b3 \\
\hline BOLD:AAD1527 & Chironomidae & Micropsectra lindrothi [18] & $\mathrm{R} / \mathrm{U} / \mathrm{C} / \mathrm{S}$ & $\mathrm{R} /-/-/-$ & 5 & 4 & & b5 b3 & 3 b5 b3 & & & b5 b3 \\
\hline BOLD:AAC7552 & Chironomidae & Micropsectra pallidula [24] & $\mathrm{R} / \mathrm{U} / \mathrm{C} / \mathrm{S}$ & $\mathrm{R} / \mathrm{U} / \mathrm{C} / \mathrm{S}$ & 91 & 53 & & b5 b3 & 3 b5 b3 & b5 b3 & b5 b3 & 3 b5 b3 \\
\hline BOLD:AAI1530 & Chironomidae & Micropsectra sp. 5ES [33] & $\mathrm{R} / \mathrm{U} / \mathrm{C} / \mathrm{S}$ & $\mathrm{R} / \mathrm{U} / \mathrm{C} / \mathrm{S}$ & 61 & 1641 & & b5 b3 & 3 b5 b3 & b5 b3 & b3 & b5 b3 \\
\hline BOLD:ACR0263 & Chironomidae & Microtendipes pedellus [8] & $R / U / C / S$ & $R / U / C / S$ & 12 & 19 & & b5 b3 & & b5 b3 & & b5 b3 \\
\hline BOLD:AAW0928 & Chironomidae & Nanocladius rectinervis [6] & $R / U / C / S$ & $R / U / C / S$ & 593 & 166 & b5 b3 & b5 b3 & 3 b5 b3 & b5 b3 & b5 b3 & 3 b5 b3 \\
\hline BOLD:AAD8971 & Chironomidae & Orthocladius oblidens [330] & $\mathrm{R} / \mathrm{U} / \mathrm{C} / \mathrm{S}$ & $\mathrm{R} / \mathrm{U} / \mathrm{C} / \mathrm{S}$ & 11359 & 5138 & b5 b3 & b5 b3 & 3 b5 b3 & b5 b3 & b5 b3 & 3 b5 b3 \\
\hline BOLD:AAM5389 & Chironomidae & Orthocladius rubicundus [119] & $\mathrm{R} / \mathrm{U} / \mathrm{C} / \mathrm{S}$ & $\mathrm{R} / \mathrm{U} / \mathrm{C} / \mathrm{S}$ & 98 & 64 & b5 & b5 b3 & 3 b5 b3 & b5 b3 & & b5 b3 \\
\hline BOLD:AAW5449 & Chironomidae & Orthocladius rubicundus [25] & $\mathrm{R} / \mathrm{U} / \mathrm{C} / \mathrm{S}$ & $\mathrm{R} / \mathrm{U} / \mathrm{C} / \mathrm{S}$ & 39 & 145 & & b5 b3 & 3 b5 b3 & b5 b3 & & b5 b3 \\
\hline BOLD:ACX3335 & Chironomidae & Paracladius quadrinodosus [2] & $-/-/-/-$ & $\mathrm{R} /-/-/-$ & 0 & 5 & & b3 & & b3 & & b3 \\
\hline BOLD:ACT5340 & Chironomidae & Paracladopelma camptolabis [2] & $\mathrm{R} / \mathrm{U} / \mathrm{C} / \mathrm{S}$ & $\mathrm{R} / \mathrm{U} / \mathrm{C} / \mathrm{S}$ & 13 & 15 & b5 b3 & b3 & b5 b3 & b3 & & b5 b3 \\
\hline BOLD:AAW4635 & Chironomidae & Paratanytarsus dissimilis [12] & $\mathrm{R} / \mathrm{U} / \mathrm{C} / \mathrm{S}$ & $\mathrm{R} / \mathrm{U} / \mathrm{C} / \mathrm{S}$ & 35 & 90 & & b5 b3 & 3 b5 b3 & & & b5 b3 \\
\hline BOLD:AAL3267 & Chironomidae & Paratanytarsus lauterborni [3] & $\mathrm{R} / \mathrm{U} / \mathrm{C} / \mathrm{S}$ & $\mathrm{R} / \mathrm{U} / \mathrm{C} / \mathrm{S}$ & 157 & 239 & & b5 b3 & 3 b5 b3 & b5 b3 & & b5 b3 \\
\hline BOLD:ACM0242 & Chironomidae & Paratanytarsus sp. & $\mathrm{R} / \mathrm{U} / \mathrm{C} / \mathrm{S}$ & $\mathrm{R} / \mathrm{U} / \mathrm{C} / \mathrm{S}$ & 47 & 4 & & b5 b3 & & b5 b3 & & b5 b3 \\
\hline BOLD:AAO1037 & Chironomidae & Paratendipes albimanus [127] & $\mathrm{R} / \mathrm{U} / \mathrm{C} / \mathrm{S}$ & $\mathrm{R} / \mathrm{U} / \mathrm{C} / \mathrm{S}$ & 161 & 159 & & b5 b3 & 3 b5 b3 & b5 b3 & b5 b3 & 3 b5 b3 \\
\hline BOLD:AAU2481 & Chironomidae & Phaenopsectra flavipes [13] & $\mathrm{R} / \mathrm{U} /-/ \mathrm{S}$ & $\mathrm{R} / \mathrm{U} / \mathrm{C} / \mathrm{S}$ & 10 & 20 & b5 b3 & b3 & & b5 b3 & b3 & b5 b3 \\
\hline BOLD:AAL0178 & Chironomidae & Polypedilum albicorne [78] & $\mathrm{R} / \mathrm{U} / \mathrm{C} / \mathrm{S}$ & $\mathrm{R} / \mathrm{U} / \mathrm{C} / \mathrm{S}$ & 145 & 104 & & b5 b3 & 3 b5 b3 & b5 b3 & b5 b3 & 3 b5 b3 \\
\hline BOLD:AAM9576 & Chironomidae & Polypedilum albinodus [3] & $R / U / C / S$ & $R / U / C / S$ & 227 & 25 & & b5 b3 & 3 b5 b3 & b5 b3 & & b5 b3 \\
\hline
\end{tabular}

This article is protected by copyright. All rights reserved. 


\begin{tabular}{|c|c|c|c|c|c|c|c|c|c|c|c|}
\hline BIN & Family & Main species id of BIN & bc-5' & bc-3' & $r-b c-5$ ' $r$ & r-bc-3' & & MC & MS & T1 & $\mathrm{T2}$ \\
\hline BOLD:AAW4728 & Chironomidae & Polypedilum pullum [2] & $\mathrm{R} /-/-/-$ & $-/-/-/-$ & 4 & 0 & & b5 & b5 & & b5 \\
\hline BOLD:AAD7458 & Chironomidae & Prodiamesa olivacea [46] & $\mathrm{R} / \mathrm{U} / \mathrm{C} / \mathrm{S}$ & $\mathrm{R} / \mathrm{U} / \mathrm{C} / \mathrm{S}$ & 79 & 16 & b5 b3 & b5 b3 b5 & b5 b3 & & b5 b3 \\
\hline BOLD:ACQ1908 & Chironomidae & Rheocricotopus chalybeatus [6] & $\mathrm{R} / \mathrm{U} / \mathrm{C} / \mathrm{S}$ & $-1-/-1-$ & 58 & 0 & & b5 & b5 & & b5 \\
\hline BOLD:AAV2322 & Chironomidae & Rheocricotopus fuscipes [20] & $\mathrm{R} / \mathrm{U} / \mathrm{C} / \mathrm{S}$ & $\mathrm{R} / \mathrm{U} / \mathrm{C} / \mathrm{S}$ & 11 & 31 & b5 b3 & & b5 b3 & & b5 b3 \\
\hline BOLD:AAD0309 & Chironomidae & Stempellina bausei [10] & $\mathrm{R} / \mathrm{U} / \mathrm{C} / \mathrm{S}$ & $\mathrm{R} / \mathrm{U} / \mathrm{C} / \mathrm{S}$ & 141 & 438 & b5 b3 & b5 & b5 b3 & b5 b3 b & b5 b3 \\
\hline BOLD:AAU2625 & Chironomidae & Stempellinella edwardsi [10] & $\mathrm{R} / \mathrm{U} /-/ \mathrm{S}$ & $\mathrm{R} / \mathrm{U} / \mathrm{C} / \mathrm{S}$ & 7 & 9 & & b5 b3 & b5 b3 & & b5 b3 \\
\hline BOLD:ACM5335 & Chironomidae & Synorthocladius semivirens [7] & $\mathrm{R} / \mathrm{U} / \mathrm{C} / \mathrm{S}$ & $\mathrm{R} / \mathrm{U} / \mathrm{C} / \mathrm{S}$ & 950 & 3924 & b5 b3 & b5 b3 b5 b3 b & b5 b3 & b5 b3 b & b5 b3 \\
\hline BOLD:ACQ8988 & Chironomidae & Tanytarsus brundini [13] & $R / U /-/ S$ & $\mathrm{R} / \mathrm{U} / \mathrm{C} / \mathrm{S}$ & 2716 & 3155 & & b5 b3 b5 b3 b & b5 b3 & b5 b3 b & b5 b3 \\
\hline BOLD:AAB9119 & Chironomidae & Tanytarsus brundini [5] & $\mathrm{R} /-/ \mathrm{C} /-$ & $\mathrm{R} /-/-/-$ & 1496 & 16 & & b5 b3 b5 b3 b & & b5 $\quad b$ & b5 b3 \\
\hline BOLD:AAW1102 & Chironomidae & Tanytarsus ejuncidus [24] & $\mathrm{R} / \mathrm{U} / \mathrm{C} /-$ & $\mathrm{R} / \mathrm{U} / \mathrm{C} / \mathrm{S}$ & 2398 & 3230 & b5 b3 & b5 b3 b5 b3 b & b5 b3 & b5 b3 b & b5 b3 \\
\hline BOLD:AAU4439 & Chironomidae & Tanytarsus eminulus [124] & $\mathrm{R} / \mathrm{U} / \mathrm{C} / \mathrm{S}$ & $\mathrm{R} / \mathrm{U} / \mathrm{C} / \mathrm{S}$ & 4847 & 2023 & b5 & b5 b3 b5 b3 b & b5 b3 & b5 b3 b & b5 b3 \\
\hline BOLD:ACF7553 & Chironomidae & Tanytarsus heusdensis [5] & $\mathrm{R} /-/-/-$ & $\mathrm{R} /-/-/-$ & 1 & 5 & & b5 b3 b & b5 b3 & b5 b3 & b3 \\
\hline BOLD:AAV3526 & Chironomidae & Tanytarsus heusdensis [6] & $\mathrm{R} / \mathrm{U} / \mathrm{C} / \mathrm{S}$ & $\mathrm{R} / \mathrm{U} / \mathrm{C} / \mathrm{S}$ & 2 & 34 & b5 b3 & b3 $b$ & b5 b3 & & b5 b3 \\
\hline BOLD:ACR3318 & Chironomidae & Tanytarsus pallidicornis [10] & $R / U / C / S$ & $\mathrm{R} / \mathrm{U} / \mathrm{C} / \mathrm{S}$ & 34 & 469 & b3 & b5 b3 b5 b3 b & b5 b3 & b3 & b5 b3 \\
\hline BOLD:ACD2995 & Empididae & Chelifera precatoria [6] & $\mathrm{R} / \mathrm{U} / \mathrm{C} / \mathrm{S}$ & $\mathrm{R} / \mathrm{U} / \mathrm{C} / \mathrm{S}$ & 156 & 385 & b5 b3 & b5 b3 $\quad b$ & b5 b3 & b5 b3 b & b5 b3 \\
\hline BOLD:ACZ6583 & Ephydridae & Scatella tenuicosta [8] & $\mathrm{R} / \mathrm{U} / \mathrm{C} / \mathrm{S}$ & $\mathrm{R} / \mathrm{U} / \mathrm{C} / \mathrm{S}$ & 27 & 13 & & b5 b3 & b5 b3 & b5 b3 & \\
\hline BOLD:ACP1316 & n.a & Diptera & $\mathrm{R} / \mathrm{U} / \mathrm{C} / \mathrm{S}$ & $\mathrm{R} / \mathrm{U} / \mathrm{C} / \mathrm{S}$ & 106 & 95 & b5 b3 & b5 b3 b5 & b5 b3 & & b5 b3 \\
\hline BOLD:ACY5064 & n.a & Diptera & $\mathrm{R} / \mathrm{U} / \mathrm{C} / \mathrm{S}$ & $\mathrm{R} / \mathrm{U} / \mathrm{C} / \mathrm{S}$ & 97 & 260 & b5 b3 & b5 b3 b5 b3 b & b5 b3 & b5 b3 b & b5 b3 \\
\hline BOLD:ABA7297 & Pediciidae & Dicranota bimaculata [6] & $\mathrm{R} /-/-/-$ & $\mathrm{R} / \mathrm{U} / \mathrm{C} / \mathrm{S}$ & 12 & 140 & b5 b3 & b5 b3 & & & b5 b3 \\
\hline BOLD:AAL7819 & Psychodidae & Psychodasp. [8] & $\mathrm{R} / \mathrm{U} / \mathrm{C} /-$ & $\mathrm{R} /-/-/-$ & 60 & 3 & & b5 b3 $\quad b$ & b5 b3 & b5 b3 & \\
\hline BOLD:AAN3314 & Simuliidae & Simulium ornatum [41] & $\mathrm{R} / \mathrm{U} / \mathrm{C} / \mathrm{S}$ & $\mathrm{R} / \mathrm{U} / \mathrm{C} / \mathrm{S}$ & 524 & 178 & b5 b3 & b5 b3 b5 b3 b & b5 b3 & b5 b3 b & b5 b3 \\
\hline BOLD:AAA8323 & Simuliidae & Simulium silvestre [151] & $\mathrm{R} /-/-/-$ & $-|-|-\mid-$ & 1 & 0 & b5 & b5 & & & b5 \\
\hline BOLD:AAP9556 & Simuliidae & Simulium velutinum [11] & $\mathrm{R} / \mathrm{U} / \mathrm{C} / \mathrm{S}$ & $\mathrm{R} / \mathrm{U} / \mathrm{C} / \mathrm{S}$ & 191 & 191 & b3 & b5 b3 b5 b3 & & b3 & b5 b3 \\
\hline BOLD:AAB8624 & Simuliidae & Simulium vernum [27] & $\mathrm{R} / \mathrm{U} / \mathrm{C} / \mathrm{S}$ & $-/-/-/-$ & 162 & 0 & b5 & b5 & & & b5 \\
\hline BOLD:AAN6407 & Sphaeroceridae & Coproica ferruginata [126] & $\mathrm{R} / \mathrm{U} / \mathrm{C} / \mathrm{S}$ & $-/-/-/-$ & 11 & 0 & b5 & & b5 & & b5 \\
\hline BOLD:AAJ5023 & Tabanidae & Chrysops caecutiens [8] & $\mathrm{R} / \mathrm{U} / \mathrm{C} / \mathrm{S}$ & $-/-/-/-$ & 18 & 0 & b5 & b5 & & & b5 \\
\hline BOLD:ABV4656 & Tipulidae & Tipula benesignata [2] & $\mathrm{R} / \mathrm{U} / \mathrm{C} / \mathrm{S}$ & $\mathrm{R} / \mathrm{U} / \mathrm{C} / \mathrm{S}$ & 21 & 26 & b5 b3 & & b5 b3 & & b5 b3 \\
\hline BOLD:AAE7386 & Tipulidae & Tipula paludosa [355] & $\mathrm{R} / \mathrm{U} / \mathrm{C} / \mathrm{S}$ & $\mathrm{R} / \mathrm{U} / \mathrm{C} / \mathrm{S}$ & 238 & 1516 & b5 b3 & & b5 b3 & & b5 b3 \\
\hline BOLD:AAF6378 & Tipulidae & Tvetenia calvescens [14] & $-/-/-1-$ & $\mathrm{R} /-/-/-$ & 0 & 4 & & b3 & b3 & b3 & \\
\hline BOLD:AAG1011 & Tipulidae & Tvetenia calvescens [186] & $R / U / C / S$ & $R / U / C / S$ & 14729 & 1930 & b5 b3 & b5 b3 b5 b3 b & b5 b3 & b5 b3 b & b5 b3 \\
\hline
\end{tabular}

Notes: bc-5': coxl barcode 5' fragment; bc-3': coxl barcode 3' fragment; R/U/C/S: Indicates detection based on Reads, USEARCH, CROP and SWARM respectively. r-bc-5' and r-bc-3': Number of reads matched for bc-5' and bc-3' respectively. C: Control (upstream) sites; I: Impacted (downstream) sites; MC: Macrofauna subsamples; MS; Meiofauna subsamples; T1: Samples collected in time 1 (11 days after the spill); T2: Samples collected in time 2 (2.5 months after the spill). b5 and b3 indicates the detection of the OTU with the bc-5' and bc-3' fragments respectively based on the processed reads. In bold species identified with indval analyses as indicative for impacted sites. Named species are the most abundant within each BIN, in brackets the number of specimens identified to species level in the reference database.

This article is protected by copyright. All rights reserved. 
Table 2. Betadiversity values, NMDS stress values and p-values for the comparison between control and impacted sites.

\begin{tabular}{|c|c|c|c|c|c|c|c|c|c|c|c|c|c|}
\hline \multirow[b]{2}{*}{ Taxa } & \multirow{2}{*}{$\begin{array}{c}\text { Dataset } \\
\text { DNA } \\
\text { fragment }\end{array}$} & \multirow[b]{2}{*}{ Method } & \multicolumn{5}{|c|}{ Betadiversity (Sorensen index) } & \multicolumn{3}{|c|}{$\begin{array}{l}\text { Turnover (Simpson } \\
\text { index) }\end{array}$} & \multicolumn{3}{|c|}{$\begin{array}{l}\text { Nestedness (Sorensen- } \\
\text { Simpson index) }\end{array}$} \\
\hline & & & $\begin{array}{c}\text { beta.so } \\
r\end{array}$ & $\begin{array}{l}\text { Adonis } \\
\text { p-value }\end{array}$ & $\begin{array}{c}\text { Adonis } \\
r^{2}\end{array}$ & stress & $\begin{array}{l}\text { envfit } p \text { - } \\
\text { value }\end{array}$ & beta.sim & stress & $\begin{array}{l}\text { envfit } \\
p \text {-value }\end{array}$ & beta.sne & stress & $\begin{array}{l}\text { envfit } p- \\
\text { value }\end{array}$ \\
\hline \multirow[t]{5}{*}{ Metazoa } & $\operatorname{cox} 1-3^{\prime}$ & de novo $3 \%$ & 0.85 & 0.007 & 0.18 & 0.086 & 0.023 & 0.763 & 0.144 & 0.179 & 0.087 & 0.067 & 0.099 \\
\hline & & de novo $10 \%$ & 0.835 & 0.011 & 0.19 & 0.075 & 0.018 & 0.736 & 0.143 & 0.054 & 0.099 & 0.053 & 0.099 \\
\hline & $\cos 1-5^{\prime}$ & de novo $3 \%$ & 0.833 & 0.004 & 0.17 & 0.122 & 0.016 & 0.743 & 0.149 & 0.089 & 0.09 & 0.042 & 0.158 \\
\hline & & de novo $10 \%$ & 0.821 & 0.005 & 0.18 & 0.12 & 0.036 & 0.731 & 0.118 & 0.144 & 0.09 & 0.035 & 0.107 \\
\hline & SSU & de novo $3 \%$ & 0.775 & 0.007 & 0.21 & 0 & 0.107 & 0.726 & 0.108 & 0.036 & 0.049 & 0.032 & 0.35 \\
\hline \multirow[t]{7}{*}{ Insecta } & $\cos 1-3^{\prime}$ & de novo $3 \%$ & 0.862 & 0.004 & 0.18 & 0.087 & 0.025 & 0.776 & 0.143 & 0.035 & 0.085 & 0.07 & 0.382 \\
\hline & & de novo $10 \%$ & 0.849 & 0.005 & 0.19 & 0.088 & 0.009 & 0.761 & 0.144 & 0.028 & 0.088 & 0.066 & 0.37 \\
\hline & & BOLD ref & 0.857 & 0.014 & 0.17 & 0.109 & 0.108 & 0.758 & 0.146 & 0.033 & 0.099 & 0.096 & 0.536 \\
\hline & $\cos 1-5^{\prime}$ & de novo $3 \%$ & 0.841 & 0.03 & 0.16 & 0.14 & 0.153 & 0.749 & 0.154 & 0.328 & 0.092 & 0.063 & 0.369 \\
\hline & & de novo $10 \%$ & 0.818 & 0.019 & 0.17 & 0.108 & 0.066 & 0.739 & 0.115 & 0.226 & 0.079 & 0.058 & 0.314 \\
\hline & & BOLD ref & 0.846 & 0.023 & 0.16 & 0.154 & 0.089 & 0.759 & 0.162 & 0.041 & 0.087 & 0.058 & 0.196 \\
\hline & SSU & de novo $3 \%$ & 0.805 & 0.037 & 0.16 & 0.173 & 0.132 & 0.739 & 0.161 & 0.057 & 0.066 & 0.059 & 0.62 \\
\hline \multirow[t]{7}{*}{ Crustacea } & $\cos 1-3^{\prime}$ & de novo $3 \%$ & 0.801 & 0.002 & 0.23 & 0.107 & 0.013 & 0.684 & 0.122 & 0.001 & 0.118 & 0.069 & 0.656 \\
\hline & & de novo $10 \%$ & 0.773 & 0.004 & 0.28 & 0.078 & 0.009 & 0.67 & 0.133 & 0.006 & 0.103 & 0.081 & 0.779 \\
\hline & & BOLD ref & 0.826 & 0.004 & 0.29 & 0.089 & 0.001 & 0.753 & 0.077 & 0.001 & 0.073 & 0.181 & 0.669 \\
\hline & $\cos 1-5^{\prime}$ & de novo $3 \%$ & 0.762 & 0.002 & 0.38 & 0.085 & 0.006 & 0.658 & 0.095 & 0.003 & 0.104 & 0.047 & 0.102 \\
\hline & & de novo $10 \%$ & 0.763 & 0.006 & 0.43 & 0.098 & 0.004 & 0.663 & 0.073 & 0.005 & 0.1 & 0.079 & 0.196 \\
\hline & & BOLD ref & 0.797 & 0.006 & 0.34 & 0.091 & 0.004 & 0.725 & 0.073 & 0.009 & 0.072 & 0.132 & 0.475 \\
\hline & SSU & de novo $3 \%$ & 0.746 & 0.005 & 0.32 & 0.057 & 0.004 & 0.675 & 0.103 & 0.011 & 0.07 & 0.076 & 0.223 \\
\hline \multirow[t]{7}{*}{ Arthropoda } & $\cos 1-3^{\prime}$ & de novo $3 \%$ & 0.845 & 0.005 & 0.19 & 0.113 & 0.011 & 0.77 & 0.136 & 0.179 & 0.075 & 0.061 & 0.26 \\
\hline & & de novo $10 \%$ & 0.832 & 0.007 & 0.20 & 0.092 & 0.017 & 0.758 & 0.135 & 0.015 & 0.074 & 0.043 & 0.317 \\
\hline & & BOLD ref & 0.856 & 0.005 & 0.19 & 0.104 & 0.113 & 0.779 & 0.128 & 0.006 & 0.077 & 0.095 & 0.569 \\
\hline & $\operatorname{cox} 1-5^{\prime}$ & de novo $3 \%$ & 0.829 & 0.005 & 0.18 & 0.142 & 0.002 & 0.746 & 0.143 & 0.003 & 0.083 & 0.064 & 0.457 \\
\hline & & de novo $10 \%$ & 0.82 & 0.002 & 0.18 & 0.139 & 0.017 & 0.75 & 0.142 & 0.013 & 0.07 & 0 & 0.236 \\
\hline & & BOLD ref & 0.843 & 0.004 & 0.18 & 0.171 & 0.006 & 0.771 & 0.164 & 0.02 & 0.072 & 0.059 & 0.242 \\
\hline & SSU & de novo $3 \%$ & 0.787 & 0.001 & 0.22 & 0.095 & 0.006 & 0.745 & 0.118 & 0.004 & 0.042 & 0.059 & 0.798 \\
\hline
\end{tabular}

Notes: de novo $3 \%$ and de novo $10 \%$ refers to OTU clustering at these threshold values. BOLD ref indicates the results from the 'taxon dependent' approach of mapping reads against OTU clusters generated from BOLD data.

This article is protected by copyright. All rights reserved. 
Table 3. Species with indicative value as identified by indval analyses based on the results of the reference database-dependent approach for the coxl gene fragments bc-5' and bc-3'.

\begin{tabular}{|c|c|c|c|c|c|c|c|c|}
\hline BIN & Class & Order & Family & Species & GENE & $\mathbf{T}$ & $\begin{array}{l}\text { Ind. } \\
\text { value }\end{array}$ & $\mathbf{P}$ \\
\hline BOLD:AAF2659 & Branchiopoda & Diplostraca & Chydoridae & Chydorus sphaericus & bc-5' & 1 & 0.6 & 0.046 \\
\hline BOLD:AAF2659 & Branchiopoda & Diplostraca & Chydoridae & Chydorus sphaericus & bc- $5^{\prime}$ & T2-I & 1 & 0.014 \\
\hline BOLD:AAP5931 & Insecta & Diptera & Chironomidae & Cricotopus bicinctus [1] & bc- $5^{\prime}$ & T2-I & 1 & 0.024 \\
\hline BOLD:ACU8677 & Insecta & Diptera & Chironomidae & Cricotopus bicinctus [1] & bc-5' & $T 2-1$ & 1 & 0.022 \\
\hline BOLD:AAI6018 & Insecta & Diptera & Chironomidae & Cricotopus bicinctus [123] & bc-3' & 1 & 0.6 & 0.048 \\
\hline BOLD:AAI6018 & Insecta & Diptera & Chironomidae & Cricotopus bicinctus [123] & bc-3' & $T 2-1$ & 1 & 0.023 \\
\hline BOLD:AAE4568 & Insecta & Diptera & Chironomidae & Eukiefferiella claripennis [185] & bc- $5^{\prime}$ & T2-I & 1 & 0.019 \\
\hline BOLD:AAO1037 & Insecta & Diptera & Chironomidae & Paratendipes albimanus [127] & bc-3' & 1 & 0.8 & 0.024 \\
\hline BOLD:AAO1037 & Insecta & Diptera & Chironomidae & Paratendipes albimanus [127] & bc-5' & 1 & 0.8 & 0.014 \\
\hline BOLD:ACQ8988 & Insecta & Diptera & Chironomidae & Tanytarsus brundini [13] & bc-3' & 1 & 0.8 & 0.02 \\
\hline BOLD:ACQ8988 & Insecta & Diptera & Chironomidae & Tanytarsus brundini [13] & bc-5' & 1 & 0.8 & 0.02 \\
\hline BOLD:AAB9119 & Insecta & Diptera & Chironomidae & Tanytarsus brundini [5] & bc-5' & 1 & 0.8 & 0.017 \\
\hline BOLD:AAW1102 & Insecta & Diptera & Chironomidae & Tanytarsus ejuncidus [24] & bc-3' & 1 & 0.75 & 0.049 \\
\hline BOLD:AAU4439 & Insecta & Diptera & Chironomidae & Tanytarsus eminulus [124] & bc-3' & 1 & 1 & 0.002 \\
\hline BOLD:AAU4439 & Insecta & Diptera & Chironomidae & Tanytarsus eminulus [124] & bc- $5^{\prime}$ & 1 & 0.86 & 0.015 \\
\hline BOLD:ACR3318 & Insecta & Diptera & Chironomidae & Tanytarsus pallidicornis [10] & bc-3' & I & 0.86 & 0.015 \\
\hline BOLD:AAU1007 & Insecta & Ephemeroptera & Baetidae & Centroptilum luteolum [17] & bc- $5^{\prime}$ & $\mathrm{T} 2$ & 1 & 0.001 \\
\hline BOLD:ACH6832 & Malacostraca & Amphipoda & Gammaridae & Gammarus pulex [11] & bc-3' & $\mathrm{C}$ & 0.83 & 0.016 \\
\hline BOLD:ACH7960 & Malacostraca & Amphipoda & Gammaridae & Gammarus nekkensis [1] & bc-5' & $\mathrm{C}$ & 1 & 0.004 \\
\hline BOLD:ACG8343 & Malacostraca & Amphipoda & Gammaridae & Gammarus fossarum [37] & bc-5' & $\mathrm{C}$ & 0.83 & 0.025 \\
\hline BOLD:AAA1971 & Malacostraca & Isopoda & Asellidae & Asellus aquaticus [85] & bc-3' & 1 & 1 & 0.004 \\
\hline BOLD:ACV6778 & Malacostraca & Isopoda & Asellidae & Asellus aquaticus [7] & bc-3' & 1 & 0.8 & 0.01 \\
\hline BOLD:AAA1971 & Malacostraca & Isopoda & Asellidae & Asellus aquaticus [85] & bc-5' & 1 & 0.86 & 0.019 \\
\hline
\end{tabular}

Notes: T: Treatment; C: Control sites, I: impacted sites; T2: collection period 2 (2.5 months after the spill); T2-I: Impacted sites at collection period 2. Named species are the most abundant within each BIN, in brackets the number of specimens identified to species level in the reference database.

This article is protected by copyright. All rights reserved. 


\section{FIGURES}

Figure 1. The flotation method for extracting meio- and macro-fauna from the original Surber samples. The figure illustrates how after flotation (A) both fractions were separated by passage (B) through a $1 \mathrm{~mm}$ metal mesh sieve that retains the macrofauna $(\mathrm{C})$, whereas a 0.45 micron sieve retains the meiofauna (D). At both sieving steps ample water was used to flush smaller items, including bacteria and other microorganisms that otherwise might also produce PCR products.

Figure 2. Shared OTUs from using alternative parameter settings of de novo OTU generation, for each of the four steps in Fig. S2. The diagrams show the proportion of shared OTUs in a list for any pair of parameter settings. Note that in most analyses the intersection of OTU lists indicates a large percentage common to all settings, except for the clustering with CROP in the $S S U$ dataset and the $\mathrm{BFC}=20$ in the denoising step.

Figure 3. Number of OTUs at 3\% similarity thresholds at various hierarchical levels. Black: Usearch; dark grey: CROP; light grey: Swarm. The clustering of OTUs with each program was started from paired reads after quality filtering using the following parameters: BFC with $s=0.5$ for read denoising, Maxee $=1$ for read filtering, and mergepairs in Usearch for read merging.

Figure 4. Total number of OTUs with the de novo generation and read mapping approaches for the two portions of coxl. The OTU count is based on BLAST+Megan for de novo generated OTUs and on the matches to the $B O L D-O T U$ reference database for the read mapping approach. Black: Usearch at $3 \%$ sequence similarity threshold; dark grey: Usearch at 10\% similarity threshold; light grey: read mapping to BOLD reference dataset.

Figure 5. NMDS ordinations for Metazoa, Arthropoda, Insecta and Crustacea based on presence/absence community matrices as obtained by read mapping against de novo generated OTUs at a $10 \%$ similarity threshold for the $b c-3$ ' gene fragment.

Figure 6. NMDS total betadiversity ordinations for Arthropoda, Insecta and Crustacea based on presence/absence community matrices as obtained by read mapping against de novo generated OTUs at $3 \%$ and $10 \%$ for the $b c-3^{\prime}$ and $b c-5^{\prime}$ gene fragments ( $\left.b c-5^{\prime} 3 \% ; b c-3^{\prime} 3 \% ; b c-5^{\prime} 10 \% ; b c-3^{\prime} 10 \%\right)$, at $3 \%$ for SSU (SSU 3\%), and by read mapping against BOLD-OTUS ( $b c-3^{\prime}$ BOLD and bc-5' BOLD)

Figure 7. NMDS ordinations for Arthropoda and the coxl-5'and coxl-3' datasets using the reference database approach with Macro (labelled with "M") and meiofauna (labelled with "m") subsamples considered independently.

This article is protected by copyright. All rights reserved. 


\section{References}

Arribas P, Andújar C, Hopkins K et al. (2016) Metabarcoding and mitochondrial metagenomics of endogean arthropods to unveil the mesofauna of the soil. Methods in Ecology and Evolution, 7, 1071-1081.

Babraham Institute (2013) FastQC: A quality control tool for high throughput sequence data. http://www.bioinformatics.babraham.ac.uk/projects/fastqc.

Baselga A (2010) Partitioning the turnover and nestedness components of beta diversity. Global Ecology and Biogeography, 19, 134-143.

Baselga A, Orme CDL (2012) betapart: an R package for the study of beta diversity. Methods in Ecology and Evolution, 3, 808-812.

Bisconti R, Canestrelli D, Tenchini R et al. (2016) Cryptic diversity and multiple origins of the widespread mayfly species group Baetis rhodani (Ephemeroptera: Baetidae) on northwestern Mediterranean islands. Ecology and Evolution, 6, 7901-7910.

Bista I, Carvalho GR, Walsh K et al. (2017) Annual time-series analysis of aqueous eDNA reveals ecologically relevant dynamics of lake ecosystem biodiversity. Nature Communications, $\mathbf{8}$, 14087.

Blaxter ML, De Ley P, Garey JR et al. (1998) A molecular evolutionary framework for the phylum Nematoda. Nature, 392, 71-75.

Blaxter M, Mann J, Chapman T et al. (2005) Defining operational taxonomic units using DNA barcode data. Philosophical transactions of the Royal Society of London. Series B, Biological Sciences, 360, 1935-1943.

Bohan DA, Vacher C, Tamaddoni-Nezhad A et al. (2017) Next-Generation global biomonitoring: largescale, automated reconstruction of ecological networks. Trends in Ecology and Evolution, 32, 477-487.

Bolger AM, Lohse M, Usadel B (2014) Trimmomatic: A flexible trimmer for Illumina sequence data. Bioinformatics, 30, 2114-2120.

Brandon-Mong G-J, Gan H-M, Sing K-W et al. (2015) DNA metabarcoding of insects and allies: an evaluation of primers and pipelines. Bulletin of Entomological Research, 105, 717-727.

This article is protected by copyright. All rights reserved. 
Burgess R (2001) An improved protocol for separating meiofauna from sediments using colloidal silica sols. Marine Ecology Progress Series, 214, 161-165.

Camargo J (1993) Macrobenthic surveys as a valuable tool for assessing freshwater quality in the Iberian Peninsula. Environ. Monit. Assess, 24, 71-90.

Chessman BC, Trayler KM, Davis JA (2002) Family- and species-level biotic indices for macroinvertebrates of wetlands on the Swan Coastal Plain, Western Australia. Marine and Freshwater Research, 53, 919-930.

Creer S, Deiner K, Frey S et al. (2016) The ecologist's field guide to sequence-based identification of biodiversity. Methods in Ecology and Evolution, 7, 1008-1018.

Creer S, Fonseca VG, Porazinska DL et al. (2010) Ultrasequencing of the meiofaunal biosphere: Practice, pitfalls and promises. Molecular Ecology, 19, 4-20.

Deagle BE, Jarman SN, Coissac E et al. (2014) DNA metabarcoding and the cytochrome c oxidase subunit I marker: not a perfect match. Biology Letters, 10, 1789-1793.

Doublet V, Raimond R, Grandjean F et al. (2012) Widespread atypical mitochondrial DNA structure in isopods (Crustacea, Peracarida) related to a constitutive heteroplasmy in terrestrial species. Genome, 55, 234-244.

Dufrêne $M$, Legendre $P$ (1997) Species assemblages and indicator species: The need for a flexible asymmetrical approach. Ecological Monographs, 67, 345-366.

Edgar RC (2013) UPARSE: highly accurate OTU sequences from microbial amplicon reads. Nature Methods, 10, 996-8.

Elbrecht V, Leese F (2015) Can DNA-based ecosystem assessments quantify species abundance? Testing primer bias and biomass-sequence relationships with an innovative metabarcoding protocol. Plos One, 10, e0130324.

Esling P, Lejzerowicz F, Pawlowski J (2015) Accurate multiplexing and filtering for high-throughput amplicon-sequencing. Nucleic Acids Research, 43, 2513-2524.

European Commission (2000) Directive 2000/60/EC of the European Parliament and of the Council establishing a framework for the Community action in the field of water policy. Official Journal (OJ L 327).

This article is protected by copyright. All rights reserved. 
Fonseca VG, Carvalho GR, Sung W et al. (2010) Second-generation environmental sequencing unmasks marine metazoan biodiversity. Nature Communications, 1, 98.

Friberg N, Bonada N, Bradley DC et al. (2011) Biomonitoring of Human Impacts in Freshwater Ecosystems. The Good, the Bad and the Ugly. Advances in Ecological Research, 44, 1-68.

Gray C, Hildrew AG, Lu X et al. (2016) Recovery and nonrecovery of freshwater food webs from the effects of acidification. Advances in Ecological Research, 55, 475-534.

Guardiola M, Uriz MJ, Taberlet P et al. (2015) Deep-sea, deep-sequencing: Metabarcoding extracellular DNA from sediments of marine canyons. PLOS ONE, 10.

Gutiérrez-Cánovas C, Velasco J, Millán A (2008) SALINDEX: A macroinvertebrate index for assessing the ecological status of saline "ramblas" from SE of the Iberian Peninsula. Limnetica, 27, 299316.

Hajibabaei M, Baird DJ, Fahner NA, Beiko R, Golding GB (2016) A new way to contemplate Darwin's tangled bank: how DNA barcodes are reconnecting biodiversity science and biomonitoring. Philosophical Transactions of the Royal Society of London B: Biological Sciences, 371, 20150330.

Hao X, Jiang R, Chen T (2011) Clustering 16S rRNA for OTU prediction: a method of unsupervised Bayesian clustering. Bioinformatics (Oxford, England), 27, 611-8.

Hebert PDN, Cywinska A, Ball SL, DeWaard JR (2003) Biological identifications through DNA barcodes. Proceedings of the Royal Society of London B, 270, 313-21.

Huson DH, Auch AF, Qi J, Schuster SC (2007) MEGAN analysis of metagenomic data. Genome Research, 17, 377-386.

Ingvason HR, Ólafsson JS, Gardarsson A (2004) Food selection of Tanytarsus gracilentus larvae (Diptera: Chironomidae): An analysis of instars and cohorts. Aquatic Ecology, 38, 231-237.

Ji Y, Ashton L, Pedley SSM et al. (2013) Reliable, verifiable and efficient monitoring of biodiversity via metabarcoding. Ecology Letters, 16, 1245-57.

Jones FC (2008) Taxonomic sufficiency: The influence of taxonomic resolution on freshwater bioassessments using benthic macroinvertebrates. Environmental Reviews, 16, 45-69.

Karaman GS, Pinkster S (1977) Freshwater Gammarus species from Europe, North Africa and adjacent regions of Asia (Crustacea-Amphipoda). Part I. Gammarus pilex-group and related species. Bijdragen tot de Dierkunde, 47, 1-97.

This article is protected by copyright. All rights reserved. 
Katoh K, Standley DM (2013) MAFFT multiple sequence alignment software version 7: Improvements in performance and usability. Molecular Biology and Evolution, 30, 772-780.

Kearse M, Moir R, Wilson A et al. (2012) Geneious Basic: An integrated and extendable desktop software platform for the organization and analysis of sequence data. Bioinformatics, 28, 1647-1649.

Lanzén A, Lekang K, Jonassen I, Thompson EM, Troedsson C (2016) High-throughput metabarcoding of eukaryotic diversity for environmental monitoring of offshore oil-drilling activities. Molecular Ecology, 25, 4392-4406.

Leese F, Altermatt F, Bouchez A et al. (2016) DNAqua-Net: Developing new genetic tools for bioassessment and monitoring of aquatic ecosystems in Europe. Research Ideas and Outcomes, 2, e11321.

Leray M, Knowlton N (2015) DNA barcoding and metabarcoding of standardized samples reveal patterns of marine benthic diversity. Proceedings of the National Academy of Sciences, 2014, 201424997.

Li H (2015) BFC: correcting Illumina sequencing errors. Bioinformatics, 1-3.

Lobo J, Shokralla S, Costa MH, Hajibabaei M, Costa FO (2015) Stepwise implementation of highthroughput sequencing metabarcoding to estuarine macrobenthic communities. Genome, $\mathbf{5 8}$, 248.

Mahé F, Rognes T, Quince C, de Vargas C, Dunthorn M (2014) Swarm: robust and fast clustering method for amplicon-based studies. PeerJ, 2, e593.

Marshall JC, Steward AL, Harch BD (2006) Taxonomic resolution and quantification of freshwater macroinvertebrate samples from an Australian dryland river: The benefits and costs of using species abundance data. Hydrobiologia, 572, 171-194.

Meyer CP, Paulay G (2005) DNA barcoding: Error rates based on comprehensive sampling. PLoS Biology, 3, e422.

Oksanen J, Blanchet FG, Kindt R et al. (2013) Vegan: Community Ecology Package. R package version 2.0-10. http://cran.r-project.org/package=vegan. $R$ package ver. 2.0-8, 254.

Paradis E, Claude J, Strimmer K (2004) APE: Analyses of phylogenetics and evolution in R language. Bioinformatics, 20, 289-290.

This article is protected by copyright. All rights reserved. 
Pauls SU, Alp M, Bálint M et al. (2014) Integrating molecular tools into freshwater ecology: Developments and opportunities. Freshwater Biology, 59, 1559-1576.

Pons J, Barraclough T, Gomez-Zurita J et al. (2006) Sequence-based species delimitation for the DNA taxonomy of undescribed insects. Systematic Biology, 55, 595-609.

Ratnasingham S, Hebert PDN (2007) BARCODING, BOLD : The Barcode of Life Data System (www.barcodinglife.org). Molecular Ecology Notes, 7, 355-364.

Ratnasingham S, Hebert PDN (2013) A DNA-based registry for all animal species: the Barcode Index Number (BIN) system. PLOS ONE, 8.

Roberts D (2007) Labdsv: Ordination and multivariate analysis for ecology. R package version 1, 3-1.

Rutschmann S, Gattolliat JL, Hughes SJ et al. (2014) Evolution and island endemism of morphologically cryptic Baetis and Cloeon species (Ephemeroptera, Baetidae) on the Canary Islands and Madeira. Freshwater Biology, 59, 2516-2527.

Sánchez-Montoya MDM, Puntí T, Suárez ML et al. (2007) Concordance between ecotypes and macroinvertebrate assemblages in Mediterranean streams. Freshwater Biology, 52, 22402255.

Schirmer M, ljaz UZ, D'Amore R et al. (2015) Insight into biases and sequencing errors for amplicon sequencing with the Illumina MiSeq platform. Nucleic Acids Research, 43, e37.

Schloss PD, Westcott SL (2011) Assessing and improving methods used in operational taxonomic unit-based approaches for $16 \mathrm{~S}$ rRNA gene sequence analysis. Applied and Environmental Microbiology, 77, 3219-3226.

Schmidt-Kloiber A, Hering D (2015) Www.freshwaterecology.info - An online tool that unifies, standardises and codifies more than 20,000 European freshwater organisms and their ecological preferences. Ecological Indicators, 53, 271-282.

Schmidt-Kloiber A, Nijboer RC (2004) The effect of taxonomic resolution on the assessment of ecological water quality classes. Hydrobiologia, 516, 269-283.

Shokralla S, Gibson JF, Nikbakht H et al. (2014) Next-generation DNA barcoding: Using nextgeneration sequencing to enhance and accelerate DNA barcode capture from single specimens. Molecular Ecology Resources, 14, 892-901.

This article is protected by copyright. All rights reserved. 
Shokralla S, Porter TM, Gibson JF et al. (2015) Massively parallel multiplex DNA sequencing for specimen identification using an Illumina MiSeq platform. Scientific reports, 5, 9687.

Staton S (2013) Pairfq: sync paired-end FASTA/Q files and keep singleton reads.

Stubauer I, Moog O (2000) Taxonomic sufficiency versus need for information- comments based on Austrian experience in biological water quality monitoring. In: Proceedings of the 27th Congress of the International Association of Theoretical and Applied Limnology. Vol. 27, p. 5.

Sworobowicz L, Grabowski M, Omasz Mamos T et al. (2015) Revisiting the phylogeography of Asellus aquaticus in Europe: insights into cryptic diversity and spatiotemporal diversification. Freshwater Biology, 60, 1824-1840.

Taberlet P, Coissac E, Pompanon F, Brochmann C, Willerslev E (2012) Towards next-generation biodiversity assessment using DNA metabarcoding. Molecular Ecology, 21, 2045-50.

Tachet H, Richoux P, Bournaud M, Usseglio-Polatera P (2002) Invertebres D'eau Douce. Systematique, Biologie, Ecologie (C Editions, Ed,). Paris.

Tang CQ, Leasi F, Obertegger U et al. (2012) The widely used small subunit 18S rDNA molecule greatly underestimates true diversity in biodiversity surveys of the meiofauna. Proceedings of the National Academy of Sciences, 109, 16208-16212.

Taylor HR, Harris WE (2012) An emergent science on the brink of irrelevance: a review of the past 8 years of DNA barcoding. Molecular Ecology Resources, 12, 377-88.

Thompson MSA, Bankier C, Bell T et al. (2016) Gene-to-ecosystem impacts of a catastrophic pesticide spill: testing a multilevel bioassessment approach in a river ecosystem. Freshwater Biology, 61, 2037-2050.

United States (1972) Federal Water Pollution Control Act Amendments of 1972. Pub.L. 92-500, October 18.

Wang Y, Naumann U, Wright ST, Warton DI (2012) Mvabund- an R package for model-based analysis of multivariate abundance data. Methods in Ecology and Evolution, 3, 471-474.

Wheeler QD (2004) Taxonomic triage and the poverty of phylogeny. Philosophical transactions of the Royal Society of London. Series B, Biological sciences, 359, 571-583.

Wickham H (2013) stringr: simple, consistent wrappers for common string operations. R package.

This article is protected by copyright. All rights reserved. 
Williams HC, Ormerod SJ, Bruford MW (2006) Molecular systematics and phylogeography of the cryptic species complex Baetis rhodani (Ephemeroptera, Baetidae). Molecular Phylogenetics and Evolution, 40, 370-382.

Woodward G, Gray C, Baird DJ (2013) Biomonitoring for the 21st Century: new perspectives in an age of globalisation and emerging environmental threats. Limnetica, 32, 159-174.

Wright JF, Sutcliffe DW, Furse MT (2000) Assessing the biological quality of fresh waters. Freshwater Biological Association, Ambleside, Cumbria, UK.

Yang C, Wang X, Miller JA et al. (2014) Using metabarcoding to ask if easily collected soil and leaflitter samples can be used as a general biodiversity indicator. Ecological Indicators, 46, 379389.

Yu D, Ji Y, Emerson B et al. (2012) Biodiversity soup: metabarcoding of arthropods for rapid biodiversity assessment and biomonitoring. Methods in Ecology and Evolution, 3, 613-623.

Zhang J, Kapli P, Pavlidis P, Stamatakis A (2013) A general species delimitation method with applications to phylogenetic placements. Bioinformatics (Oxford, England), 29, 2869-76.

Zhang J, Kobert K, Flouri T, Stamatakis A (2014) PEAR: a fast and accurate Illumina Paired-End reAd mergeR. Bioinformatics (Oxford, England), 30, 614-20.

Zinger L, Chave J, Coissac E et al. (2016) Extracellular DNA extraction is a fast, cheap and reliable alternative for multi-taxa surveys based on soil DNA. Soil Biology and Biochemistry, 96, 16-19.

Table 1. BINs of Diptera from BOLD identified based on usearch_global searches under a 3\% similarity threshold of (i) processed reads and (ii) OTUs clustered at 3\% (details in text).

\begin{tabular}{|c|c|c|c|c|c|c|c|c|c|c|c|c|}
\hline BIN & Family & Main species id of BIN & bc-5' & bc-3' & $r-b c-5$ & r-bc-3' & C & 1 & MC & MS & T1 & T2 \\
\hline BOLD:AAJ7051 & Agromyzidae & Agromyza pseudoreptans [19] & $-/-/-/-$ & $\mathrm{R} / \mathrm{U} / \mathrm{C} / \mathrm{S}$ & 0 & 5 & & b3 & b3 & & b3 & \\
\hline BOLD:ACI4790 & Bibionidae & Dilophus febrilis [21] & $R / U / C / S$ & $R / U / C / S$ & 478 & 428 & b5 b3 & & b5 b3 & b5 & b5 & b5 b3 \\
\hline BOLD:ACP0608 & Cecidomyiidae & Cecidomyiidae [3] & $\mathrm{R} / \mathrm{U} / \mathrm{C} / \mathrm{S}$ & $\mathrm{R} /-/-/ \mathrm{S}$ & 455 & 31 & & b5 b & & b5 b3 & & b5 b3 \\
\hline BOLD:ACS1169 & Ceratopogonidae & Palpomyia flavipes [6] & $\mathrm{R} / \mathrm{U} /-/-$ & $\mathrm{R} / \mathrm{U} / \mathrm{C} / \mathrm{S}$ & 20 & 41 & b5 b3 & b5 b & 3 b5 b3 & b5 b3 & b5 b3 & 3 b5 b3 \\
\hline BOLD:ACD1957 & Chironomidae & \multicolumn{2}{|c|}{ Apsectrotanypus trifascipennis $[9] \mathrm{R} / \mathrm{U} / \mathrm{C} / \mathrm{S}$} & $\mathrm{R} / \mathrm{U} / \mathrm{C} / \mathrm{S}$ & 81 & 1446 & b5 b3 & b5 b & 3 b5 b3 & b5 b3 & & b5 b3 \\
\hline BOLD:ADE2432 & Chironomidae & Brillia bifida [6] & $\mathrm{R} /-/-/-$ & $-/-/-/-$ & 1 & 0 & & b5 & & b5 & b5 & \\
\hline BOLD:ACR1089 & Chironomidae & Chironomidae & $\mathrm{R} / \mathrm{U} / \mathrm{C} / \mathrm{S}$ & $\mathrm{R} / \mathrm{U} / \mathrm{C} / \mathrm{S}$ & 550 & 93 & b5 b3 & b5 b & 3 b5 & b5 b3 & b5 b3 & $3 \mathrm{~b} 5 \mathrm{~b} 3$ \\
\hline BOLD:ACP8764 & Chironomidae & Chironomidae [13] & $\mathrm{R} / \mathrm{U} / \mathrm{C} / \mathrm{S}$ & $-1-1-1-$ & 130 & 0 & & b5 & b5 & b5 & & b5 \\
\hline BOLD:ACP6740 & Chironomidae & Chironomidae [60] & $\mathrm{R} /-/-/-$ & $\mathrm{R} / \mathrm{U} / \mathrm{C} / \mathrm{S}$ & 11 & 9 & & b5 b & 3 b3 & b5 b3 & & b5 b3 \\
\hline BOLD:ACP2182 & Chironomidae & Chironomidae [92] & $\mathrm{R} / \mathrm{U} / \mathrm{C} / \mathrm{S}$ & $\mathrm{R} / \mathrm{U} / \mathrm{C} / \mathrm{S}$ & 33 & 26 & & b5 b & & b5 b3 & & b5 b3 \\
\hline BOLD:AAW5799 & Chironomidae & Conchapelopia hittmairorum [3] & R/-/-/- & R/-/-/- & 1 & 4 & & b5 b & & b5 b3 & & b5 b3 \\
\hline BOLD:AAP5886 & Chironomidae & Conchapelopia melanops [11] & $\mathrm{R} / \mathrm{U} / \mathrm{C} / \mathrm{S}$ & $\mathrm{R} / \mathrm{U} / \mathrm{C} / \mathrm{S}$ & 1018 & 331 & b5 b3 & b5 b & 3 b5 b3 & b5 b3 & b5 b3 & 3 b5 b3 \\
\hline BOLD:ACQ3496 & Chironomidae & Conchapelopia pallidula [1] & $\mathrm{R} / \mathrm{U} / \mathrm{C} / \mathrm{S}$ & $-/-/-/-$ & 3 & 0 & & b5 & & b5 & & b5 \\
\hline BOLD:ACD1670 & Chironomidae & Corynoneura sp. & $\mathrm{R} / \mathrm{U} / \mathrm{C} / \mathrm{S}$ & $\mathrm{R} / \mathrm{U} / \mathrm{C} / \mathrm{S}$ & 1178 & 1263 & b5 b3 & b5 b & 3 b5 b3 & b5 b3 & & b5 b3 \\
\hline BOLD:ACT8698 & Chironomidae & Corynoneura sp. [6] & $\mathrm{R} / \mathrm{U} / \mathrm{C} / \mathrm{S}$ & $\mathrm{R} / \mathrm{U} / \mathrm{C} / \mathrm{S}$ & 21 & 72 & b5 b3 & b5 b & & b5 b3 & & b5 b3 \\
\hline BOLD:AAW5785 & Chironomidae & Cricotopus albiforceps [139] & $\mathrm{R} /-/-/-$ & $\mathrm{R} /-/-/-$ & 2 & 4 & b5 b3 & & & b5 b3 & & b5 b3 \\
\hline BOLD:AAF2345 & Chironomidae & Cricotopus annulator [19] & $\mathrm{R} / \mathrm{U} / \mathrm{C} / \mathrm{S}$ & $\mathrm{R} / \mathrm{U} / \mathrm{C} / \mathrm{S}$ & 30 & 14 & & b5 b & 3 b5 b3 & b5 & & b5 b3 \\
\hline BOLD:AAP5931 & Chironomidae & Cricotopus bicinctus [1] & $\mathrm{R} /-/-/-$ & $-/-\mid-/-$ & 60 & 0 & & b5 & b5 & b5 & & b5 \\
\hline BOLD:ACU8677 & Chironomidae & Cricotopus bicinctus [1] & $\mathrm{R} /-/-/-$ & $-/-/-/-$ & 5 & 0 & & b5 & b5 & b5 & & b5 \\
\hline BOLD:AAI6018 & Chironomidae & Cricotopus bicinctus [123] & $\mathrm{R} / \mathrm{U} / \mathrm{C} /-$ & $\mathrm{R} /-\mathrm{C} / \mathrm{S}$ & 5751 & 4759 & b5 & b5 b & 3 b5 b3* & *b5 b3 & b5 & b5 b3 \\
\hline BOLD:AAT9677 & Chironomidae & Cricotopus bicinctus [27] & $\mathrm{R} / \mathrm{U} /-\mathrm{S}$ & $\mathrm{R} / \mathrm{U} / \mathrm{C} /-$ & 123 & 1991 & b3 & b5 b & 3 b5 b3 & b5 b3 & & b5 b3 \\
\hline
\end{tabular}

This article is protected by copyright. All rights reserved. 


\begin{tabular}{|c|c|c|c|c|c|c|c|c|c|c|c|c|}
\hline BIN & Family & Main species id of BIN & bc-5' & bc-3' & $r-b c-5$ ' $r$ & r-bc-3' c & & 1 & MC & MS & T1 & T2 \\
\hline BOLD:AAM5377 & Chironomidae & Cricotopus rufiventris [289] & $\mathrm{R} / \mathrm{U} / \mathrm{C} / \mathrm{S}$ & $\mathrm{R} / \mathrm{U} / \mathrm{C} / \mathrm{S}$ & 8 & 44 & & b5 b & 3 b5 b3 & b3 & & b5 b3 \\
\hline BOLD:AAA5299 & Chironomidae & Cricotopus sylvestris [64] & $\mathrm{R} / \mathrm{U} / \mathrm{C} / \mathrm{S}$ & $\mathrm{R} / \mathrm{U} / \mathrm{C} / \mathrm{S}$ & 4 & 88 & & & 3 b5 b3 & & & b5 b3 \\
\hline BOLD:AAU2576 & Chironomidae & Cricotopus trifascia [5] & $R / U / C / S$ & $R / U / C / S$ & 119 & 126 & & & 3 b5 b3 & b5 b3 & & b5 b3 \\
\hline BOLD:AAE4568 & Chironomidae & Eukiefferiella claripennis [185] & $\mathrm{R} / \mathrm{U} / \mathrm{C} /-$ & $\mathrm{R} / \mathrm{U} / \mathrm{C} /-$ & 164 & 116 & & & 3 b5 b3 & b5 b3 & & b5 b3 \\
\hline BOLD:ACT0982 & Chironomidae & Heterotrissocladius sp. $2 s W[2]$ & $\mathrm{R} / \mathrm{U} / \mathrm{C} / \mathrm{S}$ & $\mathrm{R} / \mathrm{U} / \mathrm{C} / \mathrm{S}$ & 48 & 234 & b5 b3 & b5 b & 3 b5 b3 & b5 b3 & b5 b3 b & b5 b3 \\
\hline BOLD:AAX3566 & Chironomidae & Macropelopia nebulosa [13] & $\mathrm{R} / \mathrm{U} / \mathrm{C} / \mathrm{S}$ & $\mathrm{R} / \mathrm{U} / \mathrm{C} / \mathrm{S}$ & 29 & 58 & b5 b3 & b5 & b5 b3 & b5 b3 & b5 b3 & b3 \\
\hline BOLD:AAB8862 & Chironomidae & Metriocnemus eurynotus [18] & $\mathrm{R} /-/-/-$ & $\mathrm{R} / \mathrm{U} / \mathrm{C} / \mathrm{S}$ & 3 & 18 & & b5 b & & b5 b3 & b5 b3 & \\
\hline BOLD:AAD4167 & Chironomidae & Micropsectra atrofasciata [26] & $\mathrm{R} / \mathrm{U} / \mathrm{C} / \mathrm{S}$ & $\mathrm{R} / \mathrm{U} / \mathrm{C} / \mathrm{S}$ & 826 & 596 & & & 3 b5 b3 & b5 b3 & & b5 b3 \\
\hline BOLD:AAC7823 & Chironomidae & Micropsectra contracta [14] & $\mathrm{R} / \mathrm{U} / \mathrm{C} / \mathrm{S}$ & $\mathrm{R} / \mathrm{U} / \mathrm{C} / \mathrm{S}$ & 62 & 98 & & & 3 b5 b3 & b5 b3 & & b5 b3 \\
\hline BOLD:AAD1527 & Chironomidae & Micropsectra lindrothi [18] & $\mathrm{R} / \mathrm{U} / \mathrm{C} / \mathrm{S}$ & $\mathrm{R} /-/-/-$ & 5 & 4 & & & 3 b5 b3 & & & b5 b3 \\
\hline BOLD:AAC7552 & Chironomidae & Micropsectra pallidula [24] & $\mathrm{R} / \mathrm{U} / \mathrm{C} / \mathrm{S}$ & $\mathrm{R} / \mathrm{U} / \mathrm{C} / \mathrm{S}$ & 91 & 53 & & & 3 b5 b3 & b5 b3 & b5 b3 b & b5 b3 \\
\hline BOLD:AAI1530 & Chironomidae & Micropsectra sp. 5ES [33] & $R / U / C / S$ & $R / U / C / S$ & 61 & 1641 & & b5 b & 3 b5 b3 & b5 b3 & b3 & b5 b3 \\
\hline BOLD:ACR0263 & Chironomidae & Microtendipes pedellus [8] & $\mathrm{R} / \mathrm{U} / \mathrm{C} / \mathrm{S}$ & $\mathrm{R} / \mathrm{U} / \mathrm{C} / \mathrm{S}$ & 12 & 19 & & b5 b & & b5 b3 & & b5 b3 \\
\hline BOLD:AAW0928 & Chironomidae & Nanocladius rectinervis [6] & $\mathrm{R} / \mathrm{U} / \mathrm{C} / \mathrm{S}$ & $\mathrm{R} / \mathrm{U} / \mathrm{C} / \mathrm{S}$ & 593 & 166 & b5 b3 & b5 b & o b5 b3 & b5 b3 & $b 5 b$ & b5 b3 \\
\hline BOLD:AAD8971 & Chironomidae & Orthocladius oblidens [330] & $\mathrm{R} / \mathrm{U} / \mathrm{C} / \mathrm{S}$ & $\mathrm{R} / \mathrm{U} / \mathrm{C} / \mathrm{S}$ & 11359 & 5138 & b5 b3 & b5 b & 03 b5 b3 & b5 b3 & $b 5 b$ & b5 b3 \\
\hline BOLD:AAM5389 & Chironomidae & Orthocladius rubicundus [119] & $\mathrm{R} / \mathrm{U} / \mathrm{C} / \mathrm{S}$ & $\mathrm{R} / \mathrm{U} / \mathrm{C} / \mathrm{S}$ & 98 & $64 b$ & b5 & b5 b & o3 b5 b3 & b5 b3 & & b5 b3 \\
\hline BOLD:AAW5449 & Chironomidae & Orthocladius rubicundus [25] & $\mathrm{R} / \mathrm{U} / \mathrm{C} / \mathrm{S}$ & $\mathrm{R} / \mathrm{U} / \mathrm{C} / \mathrm{S}$ & 39 & 145 & & b5 b & 03 b5 b3 & b5 b3 & & b5 b3 \\
\hline BOLD:ACX3335 & Chironomidae & Paracladius quadrinodosus [2] & $-/-/-/-$ & $\mathrm{R} /-/-/-$ & 0 & 5 & & b3 & & b3 & & b3 \\
\hline BOLD:ACT5340 & Chironomidae & Paracladopelma camptolabis [2] & $\mathrm{R} / \mathrm{U} / \mathrm{C} / \mathrm{S}$ & $\mathrm{R} / \mathrm{U} / \mathrm{C} / \mathrm{S}$ & 13 & 15 & b5 b3 & b3 & b5 b3 & b3 & & b5 b3 \\
\hline BOLD:AAW4635 & Chironomidae & Paratanytarsus dissimilis [12] & $\mathrm{R} / \mathrm{U} / \mathrm{C} / \mathrm{S}$ & $\mathrm{R} / \mathrm{U} / \mathrm{C} / \mathrm{S}$ & 35 & 90 & & b5 b & 03 b5 b3 & & & b5 b3 \\
\hline BOLD:AAL3267 & Chironomidae & Paratanytarsus lauterborni [3] & $\mathrm{R} / \mathrm{U} / \mathrm{C} / \mathrm{S}$ & $\mathrm{R} / \mathrm{U} / \mathrm{C} / \mathrm{S}$ & 157 & 239 & & b5 b & o3 b5 b3 & b5 b3 & & b5 b3 \\
\hline BOLD:ACM0242 & Chironomidae & Paratanytarsus sp. & $\mathrm{R} / \mathrm{U} / \mathrm{C} / \mathrm{S}$ & $\mathrm{R} / \mathrm{U} / \mathrm{C} / \mathrm{S}$ & 47 & 4 & & b5 b & & b5 b3 & & b5 b3 \\
\hline BOLD:AAO1037 & Chironomidae & Paratendipes albimanus [127] & $\mathrm{R} / \mathrm{U} / \mathrm{C} / \mathrm{S}$ & $\mathrm{R} / \mathrm{U} / \mathrm{C} / \mathrm{S}$ & 161 & 159 & & b5 b & b3 b5 b3 & b5 b3 & b5 b3 b & b5 b3 \\
\hline BOLD:AAU2481 & Chironomidae & Phaenopsectra flavipes [13] & $\mathrm{R} / \mathrm{U} /-/ \mathrm{S}$ & $\mathrm{R} / \mathrm{U} / \mathrm{C} / \mathrm{S}$ & 10 & 20 & b5 b3 & b3 & & b5 b3 & b3 & b5 b3 \\
\hline BOLD:AAL0178 & Chironomidae & Polypedilum albicorne [78] & $\mathrm{R} / \mathrm{U} / \mathrm{C} / \mathrm{S}$ & $\mathrm{R} / \mathrm{U} / \mathrm{C} / \mathrm{S}$ & 145 & 104 & & b5 b & 03 b5 b3 & b5 b3 & b5 b3 b & b5 b3 \\
\hline BOLD:AAM9576 & Chironomidae & Polypedilum albinodus [3] & $\mathrm{R} / \mathrm{U} / \mathrm{C} / \mathrm{S}$ & $\mathrm{R} / \mathrm{U} / \mathrm{C} / \mathrm{S}$ & 227 & 25 & & b5 b & 03 b5 b3 & b5 b3 & & b5 b3 \\
\hline BOLD:AAW4728 & Chironomidae & Polypedilum pullum [2] & $\mathrm{R} /-/-/-$ & $-/-/-1-$ & 4 & 0 & & b5 & & b5 & & b5 \\
\hline BOLD:AAD7458 & Chironomidae & Prodiamesa olivacea [46] & $\mathrm{R} / \mathrm{U} / \mathrm{C} / \mathrm{S}$ & $\mathrm{R} / \mathrm{U} / \mathrm{C} / \mathrm{S}$ & 79 & 16 & b5 b3 & b5 b & o3 b5 & b5 b3 & & b5 b3 \\
\hline BOLD:ACQ1908 & Chironomidae & Rheocricotopus chalybeatus [6] & $\mathrm{R} / \mathrm{U} / \mathrm{C} / \mathrm{S}$ & $-1-/-1-$ & 58 & 0 & & b5 & & b5 & & b5 \\
\hline BOLD:AAV2322 & Chironomidae & Rheocricotopus fuscipes [20] & $\mathrm{R} / \mathrm{U} / \mathrm{C} / \mathrm{S}$ & $\mathrm{R} / \mathrm{U} / \mathrm{C} / \mathrm{S}$ & 11 & 31 & b5 b3 & & & b5 b3 & & b5 b3 \\
\hline BOLD:AAD0309 & Chironomidae & Stempellina bausei $[10]$ & $\mathrm{R} / \mathrm{U} / \mathrm{C} / \mathrm{S}$ & $\mathrm{R} / \mathrm{U} / \mathrm{C} / \mathrm{S}$ & 141 & 438 & b5 b3 & & b5 & b5 b3 & b5 b3 b & b5 b3 \\
\hline BOLD:AAU2625 & Chironomidae & Stempellinella edwardsi [10] & $\mathrm{R} / \mathrm{U} /-/ \mathrm{S}$ & $\mathrm{R} / \mathrm{U} / \mathrm{C} / \mathrm{S}$ & 7 & 9 & & b5 b & & b5 b3 & & b5 b3 \\
\hline BOLD:ACM5335 & Chironomidae & Synorthocladius semivirens [7] & $\mathrm{R} / \mathrm{U} / \mathrm{C} / \mathrm{S}$ & $\mathrm{R} / \mathrm{U} / \mathrm{C} / \mathrm{S}$ & 950 & $3924 b$ & b5 b3 & b5 b & o3 b5 b3 & b5 b3 & b5 b3 b & b5 b3 \\
\hline BOLD:ACQ8988 & Chironomidae & Tanytarsus brundini [13] & $\mathrm{R} / \mathrm{U} /-/ \mathrm{S}$ & $\mathrm{R} / \mathrm{U} / \mathrm{C} / \mathrm{S}$ & 2716 & 3155 & & b5 b & b3 b5 b3 & b5 b3 & b5 b & b5 b3 \\
\hline BOLD:AAB9119 & Chironomidae & Tanytarsus brundini [5] & $\mathrm{R} /-/ \mathrm{C} /-$ & $\mathrm{R} /-/-/-$ & 1496 & 16 & & b5 b & b3 b5 b3 & b5 & b5 & b5 b3 \\
\hline BOLD:AAW1102 & Chironomidae & Tanytarsus ejuncidus [24] & $R / U / C /-$ & $\mathrm{R} / \mathrm{U} / \mathrm{C} / \mathrm{S}$ & 2398 & 3230 & b5 b3 & b5 b & b3 b5 b3 & b5 b3 & b5 b3 b & b5 b3 \\
\hline BOLD:AAU4439 & Chironomidae & Tanytarsus eminulus [124] & $\mathrm{R} / \mathrm{U} / \mathrm{C} / \mathrm{S}$ & $\mathrm{R} / \mathrm{U} / \mathrm{C} / \mathrm{S}$ & 4847 & 2023 & b5 & b5 b & b3 b5 b3 & b5 b3 & b5 b3 b & b5 b3 \\
\hline BOLD:ACF7553 & Chironomidae & Tanytarsus heusdensis [5] & $\mathrm{R} /-/-/-$ & $\mathrm{R} /-/-/-$ & 1 & 5 & & b5 b & & b5 b3 & b5 b3 & b3 \\
\hline BOLD:AAV3526 & Chironomidae & Tanytarsus heusdensis [6] & $\mathrm{R} / \mathrm{U} / \mathrm{C} / \mathrm{S}$ & $\mathrm{R} / \mathrm{U} / \mathrm{C} / \mathrm{S}$ & 2 & 34 & b5 b3 & & b3 & b5 b3 & & b5 b3 \\
\hline BOLD:ACR3318 & Chironomidae & Tanytarsus pallidicornis [10] & $R / U / C / S$ & $\mathrm{R} / \mathrm{U} / \mathrm{C} / \mathrm{S}$ & 34 & 469 & b3 & b5 b & b3 b5 b3 & b5 b3 & b3 & b5 b3 \\
\hline BOLD:ACD2995 & Empididae & Chelifera precatoria [6] & $\mathrm{R} / \mathrm{U} / \mathrm{C} / \mathrm{S}$ & $\mathrm{R} / \mathrm{U} / \mathrm{C} / \mathrm{S}$ & 156 & 385 & b5 b3 & b5 b & & b5 b3 & b5 b3 b & b5 b3 \\
\hline BOLD:ACZ6583 & Ephydridae & Scatella tenuicosta [8] & $\mathrm{R} / \mathrm{U} / \mathrm{C} / \mathrm{S}$ & $\mathrm{R} / \mathrm{U} / \mathrm{C} / \mathrm{S}$ & 27 & 13 & & & & b5 b3 & b5 b3 & \\
\hline BOLD:ACP1316 & n.a & Diptera & $\mathrm{R} / \mathrm{U} / \mathrm{C} / \mathrm{S}$ & $\mathrm{R} / \mathrm{U} / \mathrm{C} / \mathrm{S}$ & 106 & 95 & b5 b3 & b5 b & b3 b5 & b5 b3 & & b5 b3 \\
\hline BOLD:ACY5064 & n.a & Diptera & $\mathrm{R} / \mathrm{U} / \mathrm{C} / \mathrm{S}$ & $\mathrm{R} / \mathrm{U} / \mathrm{C} / \mathrm{S}$ & 97 & 260 & b5 b3 & b5 b & b3 b5 b3 & b5 b3 & b5 b3 b & b5 b3 \\
\hline BOLD:ABA7297 & Pediciidae & Dicranota bimaculata [6] & $\mathrm{R} /-/-/-$ & $\mathrm{R} / \mathrm{U} / \mathrm{C} / \mathrm{S}$ & 12 & 140 & b5 b3 & & b5 b3 & & & b5 b3 \\
\hline BOLD:AAL7819 & Psychodidae & Psychoda sp. [8] & $\mathrm{R} / \mathrm{U} / \mathrm{C} /-$ & $\mathrm{R} /-/-/-$ & 60 & 3 & & b5 b & & b5 b3 & b5 b3 & \\
\hline BOLD:AAN3314 & Simuliidae & Simulium ornatum [41] & $R / U / C / S$ & $\mathrm{R} / \mathrm{U} / \mathrm{C} / \mathrm{S}$ & 524 & 178 & b5 b3 & b5 b & b3 b5 b3 & b5 b3 & b5 b3 b & b5 b3 \\
\hline BOLD:AAA8323 & Simuliidae & Simulium silvestre [151] & $\mathrm{R} /-/-/-$ & $-|-|-\mid-$ & 1 & 0 & b5 & & b5 & & & b5 \\
\hline BOLD:AAP9556 & Simuliidae & Simulium velutinum [11] & $\mathrm{R} / \mathrm{U} / \mathrm{C} / \mathrm{S}$ & $\mathrm{R} / \mathrm{U} / \mathrm{C} / \mathrm{S}$ & 191 & 191 & b3 & b5 b & b3 b5 b3 & & b3 & b5 b3 \\
\hline BOLD:AAB8624 & Simuliidae & Simulium vernum [27] & $\mathrm{R} / \mathrm{U} / \mathrm{C} / \mathrm{S}$ & $-/-/-/-$ & 162 & 0 & b5 & & b5 & & & b5 \\
\hline BOLD:AAN6407 & Sphaeroceridae & Coproica ferruginata [126] & $\mathrm{R} / \mathrm{U} / \mathrm{C} / \mathrm{S}$ & $-1-1-1-$ & 11 & 0 & b5 & & & b5 & & b5 \\
\hline BOLD:AAJ5023 & Tabanidae & Chrysops caecutiens [8] & $\mathrm{R} / \mathrm{U} / \mathrm{C} / \mathrm{S}$ & $-/-/-/-$ & 18 & 0 & b5 & & b5 & & & b5 \\
\hline BOLD:ABV4656 & Tipulidae & Tipula benesignata [2] & $\mathrm{R} / \mathrm{U} / \mathrm{C} / \mathrm{S}$ & $\mathrm{R} / \mathrm{U} / \mathrm{C} / \mathrm{S}$ & 21 & 26 & b5 b3 & & & b5 b3 & & b5 b3 \\
\hline BOLD:AAE7386 & Tipulidae & Tipula paludosa [355] & $\mathrm{R} / \mathrm{U} / \mathrm{C} / \mathrm{S}$ & $\mathrm{R} / \mathrm{U} / \mathrm{C} / \mathrm{S}$ & 238 & 1516 & b5 b3 & & & b5 b3 & & b5 b3 \\
\hline BOLD:AAF6378 & Tipulidae & Tvetenia calvescens [14] & $-|-|-\mid-$ & $R /-/-/-$ & 0 & 4 & & b3 & & b3 & b3 & \\
\hline BOLD:AAG1011 & Tipulidae & Tvetenia calvescens [186] & $\mathrm{R} / \mathrm{U} / \mathrm{C} / \mathrm{S}$ & $\mathrm{R} / \mathrm{U} / \mathrm{C} / \mathrm{S}$ & 14729 & 1930 & b5 b3 & b5 b & b3 b5 b3 & b5 b3 & b5 b3 b & b5 b3 \\
\hline
\end{tabular}

Notes: bc-5': coxl barcode 5' fragment; bc-3': coxl barcode 3' fragment; R/U/C/S: Indicates detection based on Reads, USEARCH, CROP and SWARM respectively. r-bc-5' and r-bc-3': Number of reads matched for bc-5' and bc-3' respectively. C: Control (upstream) sites; I: Impacted (downstream) sites; MC: Macrofauna subsamples; MS; Meiofauna subsamples; T1: Samples collected in time 1 (11 days after the spill); T2: Samples collected in time 2 (2.5 months after the spill). b5 and b3 indicates the detection of the OTU with the bc-5' and bc-3' fragments respectively based on the processed reads. In bold species identified with indval analyses as indicative for impacted sites. Named species are the most abundant within each BIN, in brackets the number of specimens identified to species level in the reference database.

This article is protected by copyright. All rights reserved. 
Table 2. Betadiversity values, NMDS stress values and p-values for the comparison between control and impacted sites.

\begin{tabular}{|c|c|c|c|c|c|c|c|c|c|c|c|c|c|}
\hline \multirow[b]{2}{*}{ Taxa } & \multirow{2}{*}{$\begin{array}{c}\text { Dataset } \\
\text { DNA } \\
\text { fragment }\end{array}$} & \multirow[b]{2}{*}{ Method } & \multicolumn{5}{|c|}{ Betadiversity (Sorensen index) } & \multicolumn{3}{|c|}{$\begin{array}{l}\text { Turnover (Simpson } \\
\text { index) }\end{array}$} & \multicolumn{3}{|c|}{$\begin{array}{l}\text { Nestedness (Sorensen- } \\
\text { Simpson index) }\end{array}$} \\
\hline & & & $\begin{array}{c}\text { beta.so } \\
r\end{array}$ & $\begin{array}{l}\text { Adonis } \\
\text { p-value }\end{array}$ & $\begin{array}{c}\text { Adonis } \\
r^{2}\end{array}$ & stress & $\begin{array}{l}\text { envfit } p \text { - } \\
\text { value }\end{array}$ & beta.sim & stress & $\begin{array}{l}\text { envfit } \\
p \text {-value }\end{array}$ & beta.sne & stress & $\begin{array}{l}\text { envfit } p- \\
\text { value }\end{array}$ \\
\hline \multirow[t]{5}{*}{ Metazoa } & $\operatorname{cox} 1-3^{\prime}$ & de novo $3 \%$ & 0.85 & 0.007 & 0.18 & 0.086 & 0.023 & 0.763 & 0.144 & 0.179 & 0.087 & 0.067 & 0.099 \\
\hline & & de novo $10 \%$ & 0.835 & 0.011 & 0.19 & 0.075 & 0.018 & 0.736 & 0.143 & 0.054 & 0.099 & 0.053 & 0.099 \\
\hline & $\cos 1-5^{\prime}$ & de novo $3 \%$ & 0.833 & 0.004 & 0.17 & 0.122 & 0.016 & 0.743 & 0.149 & 0.089 & 0.09 & 0.042 & 0.158 \\
\hline & & de novo $10 \%$ & 0.821 & 0.005 & 0.18 & 0.12 & 0.036 & 0.731 & 0.118 & 0.144 & 0.09 & 0.035 & 0.107 \\
\hline & SSU & de novo $3 \%$ & 0.775 & 0.007 & 0.21 & 0 & 0.107 & 0.726 & 0.108 & 0.036 & 0.049 & 0.032 & 0.35 \\
\hline \multirow[t]{7}{*}{ Insecta } & $\cos 1-3^{\prime}$ & de novo $3 \%$ & 0.862 & 0.004 & 0.18 & 0.087 & 0.025 & 0.776 & 0.143 & 0.035 & 0.085 & 0.07 & 0.382 \\
\hline & & de novo $10 \%$ & 0.849 & 0.005 & 0.19 & 0.088 & 0.009 & 0.761 & 0.144 & 0.028 & 0.088 & 0.066 & 0.37 \\
\hline & & BOLD ref & 0.857 & 0.014 & 0.17 & 0.109 & 0.108 & 0.758 & 0.146 & 0.033 & 0.099 & 0.096 & 0.536 \\
\hline & $\cos 1-5^{\prime}$ & de novo $3 \%$ & 0.841 & 0.03 & 0.16 & 0.14 & 0.153 & 0.749 & 0.154 & 0.328 & 0.092 & 0.063 & 0.369 \\
\hline & & de novo $10 \%$ & 0.818 & 0.019 & 0.17 & 0.108 & 0.066 & 0.739 & 0.115 & 0.226 & 0.079 & 0.058 & 0.314 \\
\hline & & BOLD ref & 0.846 & 0.023 & 0.16 & 0.154 & 0.089 & 0.759 & 0.162 & 0.041 & 0.087 & 0.058 & 0.196 \\
\hline & SSU & de novo $3 \%$ & 0.805 & 0.037 & 0.16 & 0.173 & 0.132 & 0.739 & 0.161 & 0.057 & 0.066 & 0.059 & 0.62 \\
\hline \multirow[t]{7}{*}{ Crustacea } & $\cos 1-3^{\prime}$ & de novo $3 \%$ & 0.801 & 0.002 & 0.23 & 0.107 & 0.013 & 0.684 & 0.122 & 0.001 & 0.118 & 0.069 & 0.656 \\
\hline & & de novo $10 \%$ & 0.773 & 0.004 & 0.28 & 0.078 & 0.009 & 0.67 & 0.133 & 0.006 & 0.103 & 0.081 & 0.779 \\
\hline & & BOLD ref & 0.826 & 0.004 & 0.29 & 0.089 & 0.001 & 0.753 & 0.077 & 0.001 & 0.073 & 0.181 & 0.669 \\
\hline & $\cos 1-5^{\prime}$ & de novo $3 \%$ & 0.762 & 0.002 & 0.38 & 0.085 & 0.006 & 0.658 & 0.095 & 0.003 & 0.104 & 0.047 & 0.102 \\
\hline & & de novo $10 \%$ & 0.763 & 0.006 & 0.43 & 0.098 & 0.004 & 0.663 & 0.073 & 0.005 & 0.1 & 0.079 & 0.196 \\
\hline & & BOLD ref & 0.797 & 0.006 & 0.34 & 0.091 & 0.004 & 0.725 & 0.073 & 0.009 & 0.072 & 0.132 & 0.475 \\
\hline & SSU & de novo $3 \%$ & 0.746 & 0.005 & 0.32 & 0.057 & 0.004 & 0.675 & 0.103 & 0.011 & 0.07 & 0.076 & 0.223 \\
\hline \multirow[t]{7}{*}{ Arthropoda } & $\cos 1-3^{\prime}$ & de novo $3 \%$ & 0.845 & 0.005 & 0.19 & 0.113 & 0.011 & 0.77 & 0.136 & 0.179 & 0.075 & 0.061 & 0.26 \\
\hline & & de novo $10 \%$ & 0.832 & 0.007 & 0.20 & 0.092 & 0.017 & 0.758 & 0.135 & 0.015 & 0.074 & 0.043 & 0.317 \\
\hline & & BOLD ref & 0.856 & 0.005 & 0.19 & 0.104 & 0.113 & 0.779 & 0.128 & 0.006 & 0.077 & 0.095 & 0.569 \\
\hline & $\operatorname{cox} 1-5^{\prime}$ & de novo $3 \%$ & 0.829 & 0.005 & 0.18 & 0.142 & 0.002 & 0.746 & 0.143 & 0.003 & 0.083 & 0.064 & 0.457 \\
\hline & & de novo $10 \%$ & 0.82 & 0.002 & 0.18 & 0.139 & 0.017 & 0.75 & 0.142 & 0.013 & 0.07 & 0 & 0.236 \\
\hline & & BOLD ref & 0.843 & 0.004 & 0.18 & 0.171 & 0.006 & 0.771 & 0.164 & 0.02 & 0.072 & 0.059 & 0.242 \\
\hline & SSU & de novo $3 \%$ & 0.787 & 0.001 & 0.22 & 0.095 & 0.006 & 0.745 & 0.118 & 0.004 & 0.042 & 0.059 & 0.798 \\
\hline
\end{tabular}

Notes: de novo $3 \%$ and de novo $10 \%$ refers to OTU clustering at these threshold values. BOLD ref indicates the results from the 'taxon dependent' approach of mapping reads against OTU clusters generated from BOLD data.

This article is protected by copyright. All rights reserved. 
Table 3. Species with indicative value as identified by indval analyses based on the results of the reference database-dependent approach for the coxl gene fragments bc-5' and bc-3'.

\begin{tabular}{|c|c|c|c|c|c|c|c|c|}
\hline BIN & Class & Order & Family & Species & GENE & $\mathbf{T}$ & $\begin{array}{l}\text { Ind. } \\
\text { value }\end{array}$ & $\mathbf{P}$ \\
\hline BOLD:AAF2659 & Branchiopoda & Diplostraca & Chydoridae & Chydorus sphaericus & bc-5' & $\mathrm{I}$ & 0.6 & 0.046 \\
\hline BOLD:AAF2659 & Branchiopoda & Diplostraca & Chydoridae & Chydorus sphaericus & bc-5' & T2-I & 1 & 0.014 \\
\hline BOLD:AAP5931 & Insecta & Diptera & Chironomidae & Cricotopus bicinctus [1] & $b c-5^{\prime}$ & T2-I & 1 & 0.024 \\
\hline BOLD:ACU8677 & Insecta & Diptera & Chironomidae & Cricotopus bicinctus [1] & bc-5' & T2-I & 1 & 0.022 \\
\hline BOLD:AAI6018 & Insecta & Diptera & Chironomidae & Cricotopus bicinctus [123] & bc-3' & 1 & 0.6 & 0.048 \\
\hline BOLD:AAI6018 & Insecta & Diptera & Chironomidae & Cricotopus bicinctus [123] & bc- $3^{\prime}$ & T2-I & 1 & 0.023 \\
\hline BOLD:AAE4568 & Insecta & Diptera & Chironomidae & Eukiefferiella claripennis [185] & bc- $5^{\prime}$ & T2-I & 1 & 0.019 \\
\hline BOLD:AA01037 & Insecta & Diptera & Chironomidae & Paratendipes albimanus [127] & bc-3' & 1 & 0.8 & 0.024 \\
\hline BOLD:AAO1037 & Insecta & Diptera & Chironomidae & Paratendipes albimanus [127] & bc-5' & 1 & 0.8 & 0.014 \\
\hline BOLD:ACQ8988 & Insecta & Diptera & Chironomidae & Tanytarsus brundini [13] & bc-3' & 1 & 0.8 & 0.02 \\
\hline BOLD:ACQ8988 & Insecta & Diptera & Chironomidae & Tanytarsus brundini [13] & bc-5' & 1 & 0.8 & 0.02 \\
\hline BOLD:AAB9119 & Insecta & Diptera & Chironomidae & Tanytarsus brundini [5] & bc-5' & 1 & 0.8 & 0.017 \\
\hline BOLD:AAW1102 & Insecta & Diptera & Chironomidae & Tanytarsus ejuncidus [24] & bc-3' & 1 & 0.75 & 0.049 \\
\hline BOLD:AAU4439 & Insecta & Diptera & Chironomidae & Tanytarsus eminulus [124] & bc-3' & 1 & 1 & 0.002 \\
\hline BOLD:AAU4439 & Insecta & Diptera & Chironomidae & Tanytarsus eminulus [124] & bc- $5^{\prime}$ & 1 & 0.86 & 0.015 \\
\hline BOLD:ACR3318 & Insecta & Diptera & Chironomidae & Tanytarsus pallidicornis [10] & bc-3' & I & 0.86 & 0.015 \\
\hline BOLD:AAU1007 & Insecta & Ephemeroptera & Baetidae & Centroptilum luteolum [17] & bc- $5^{\prime}$ & $\mathrm{T} 2$ & 1 & 0.001 \\
\hline BOLD:ACH6832 & Malacostraca & Amphipoda & Gammaridae & Gammarus pulex [11] & bc-3' & $\mathrm{C}$ & 0.83 & 0.016 \\
\hline BOLD:ACH7960 & Malacostraca & Amphipoda & Gammaridae & Gammarus nekkensis [1] & bc-5' & $\mathrm{C}$ & 1 & 0.004 \\
\hline BOLD:ACG8343 & Malacostraca & Amphipoda & Gammaridae & Gammarus fossarum [37] & bc-5' & C & 0.83 & 0.025 \\
\hline BOLD:AAA1971 & Malacostraca & Isopoda & Asellidae & Asellus aquaticus [85] & bc-3' & 1 & 1 & 0.004 \\
\hline BOLD:ACV6778 & Malacostraca & Isopoda & Asellidae & Asellus aquaticus [7] & bc-3' & 1 & 0.8 & 0.01 \\
\hline BOLD:AAA1971 & Malacostraca & Isopoda & Asellidae & Asellus aquaticus [85] & $b c-5^{\prime}$ & 1 & 0.86 & 0.019 \\
\hline
\end{tabular}

Notes: T: Treatment; C: Control sites, I: impacted sites; T2: collection period 2 (2.5 months after the spill); T2-I: Impacted sites at collection period 2. Named species are the most abundant within each BIN, in brackets the number of specimens identified to species level in the reference database.

This article is protected by copyright. All rights reserved. 
A)

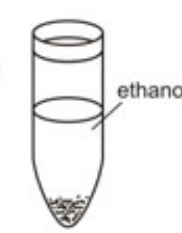

Arthropod

bulk

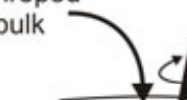

目
B)

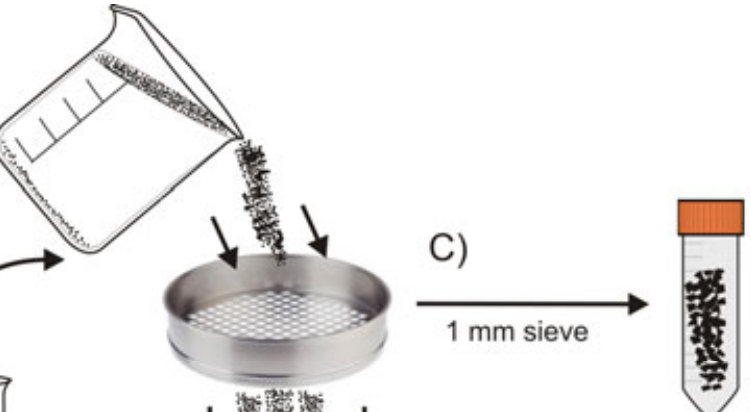

Clean macrofauna bulk

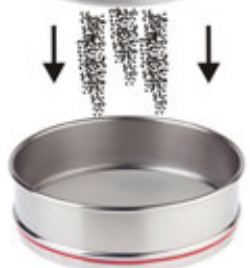

D)

$\underset{0.45 \mu \mathrm{m} \text { sieve }}{\longrightarrow}$

$+$

Ludox flotation

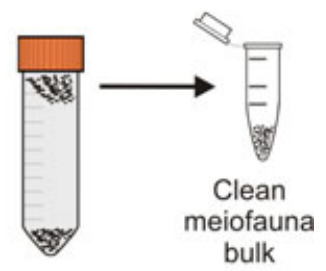

This article is protected by copyright. All rights reserved. 
Cox1_BC_5'

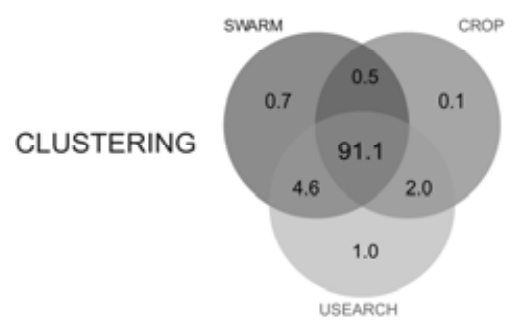

$B F C s=2 \quad B F C s=20$
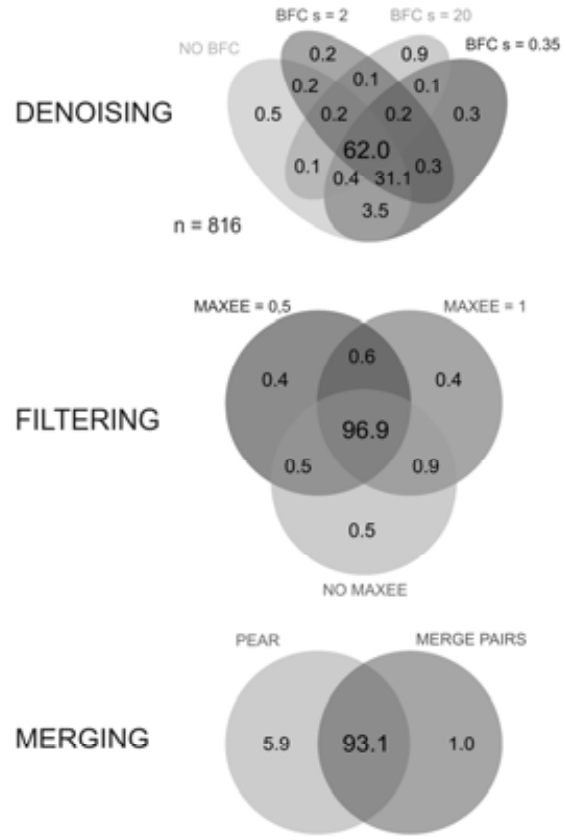

Cox1_BC_3'
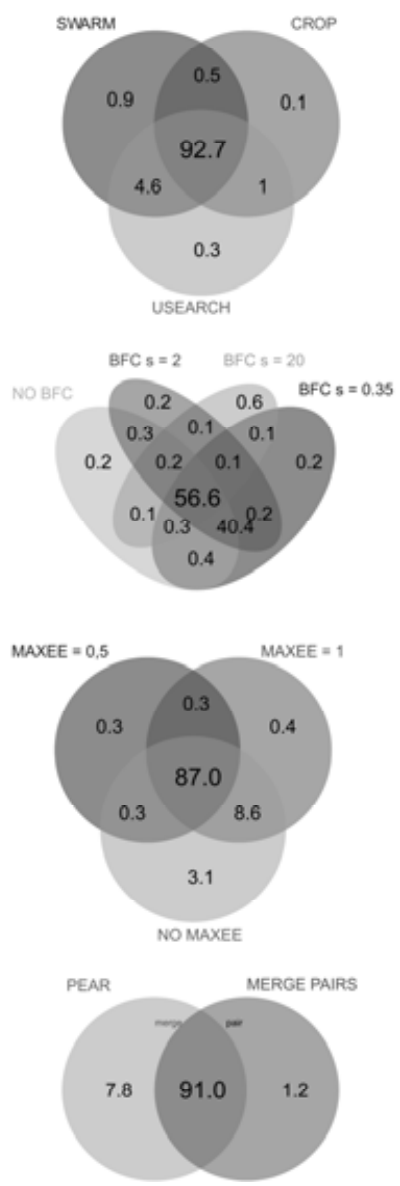

SSU
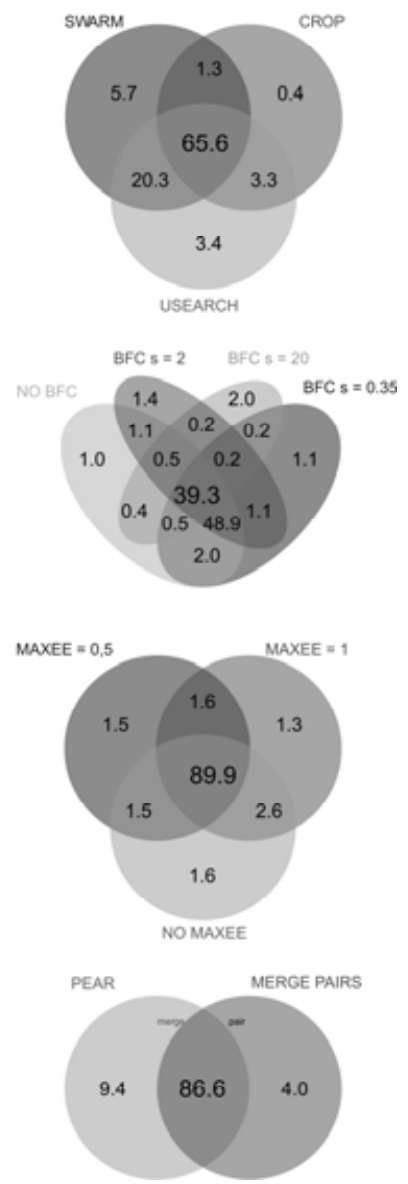

This article is protected by copyright. All rights reserved. 

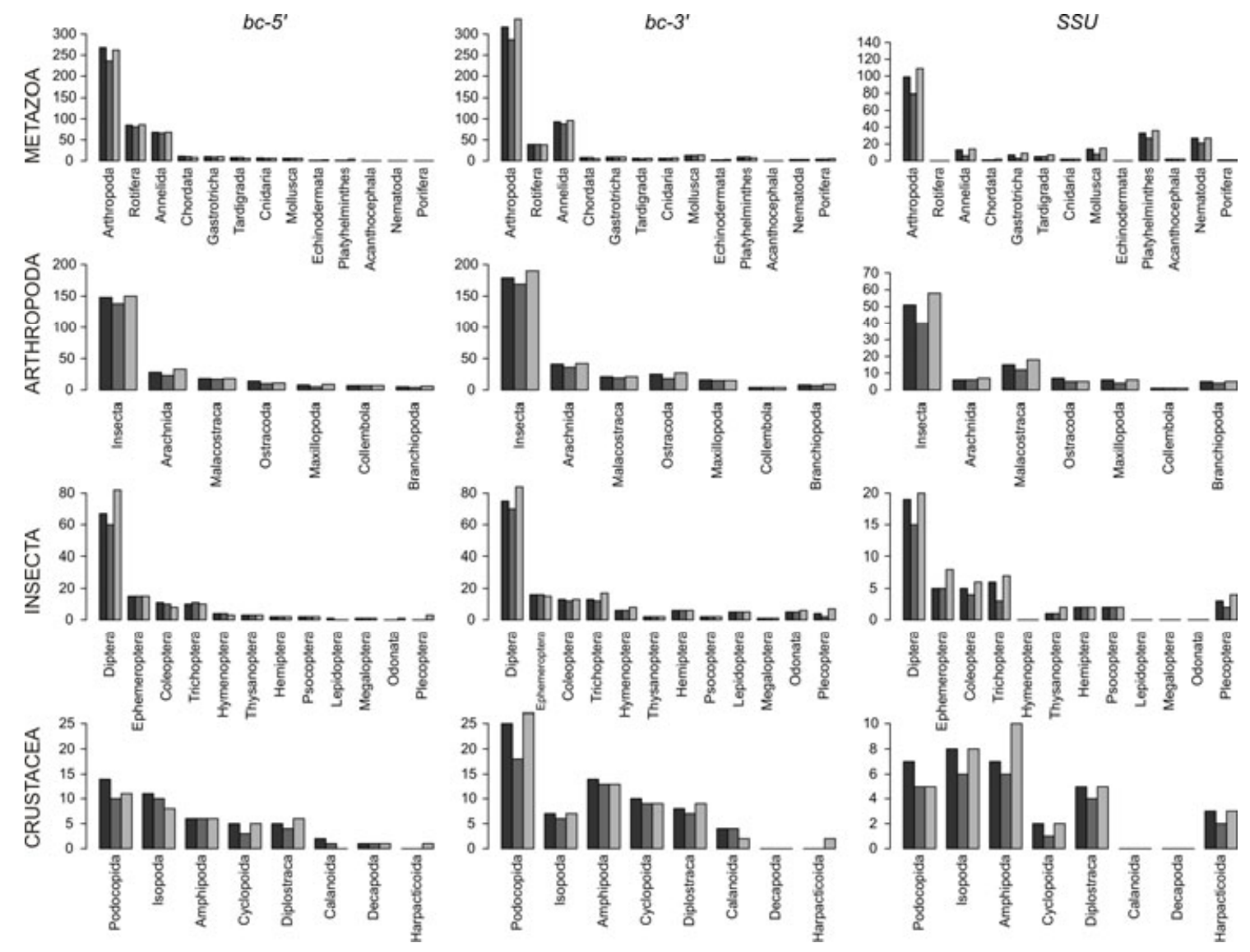

This article is protected by copyright. All rights reserved. 


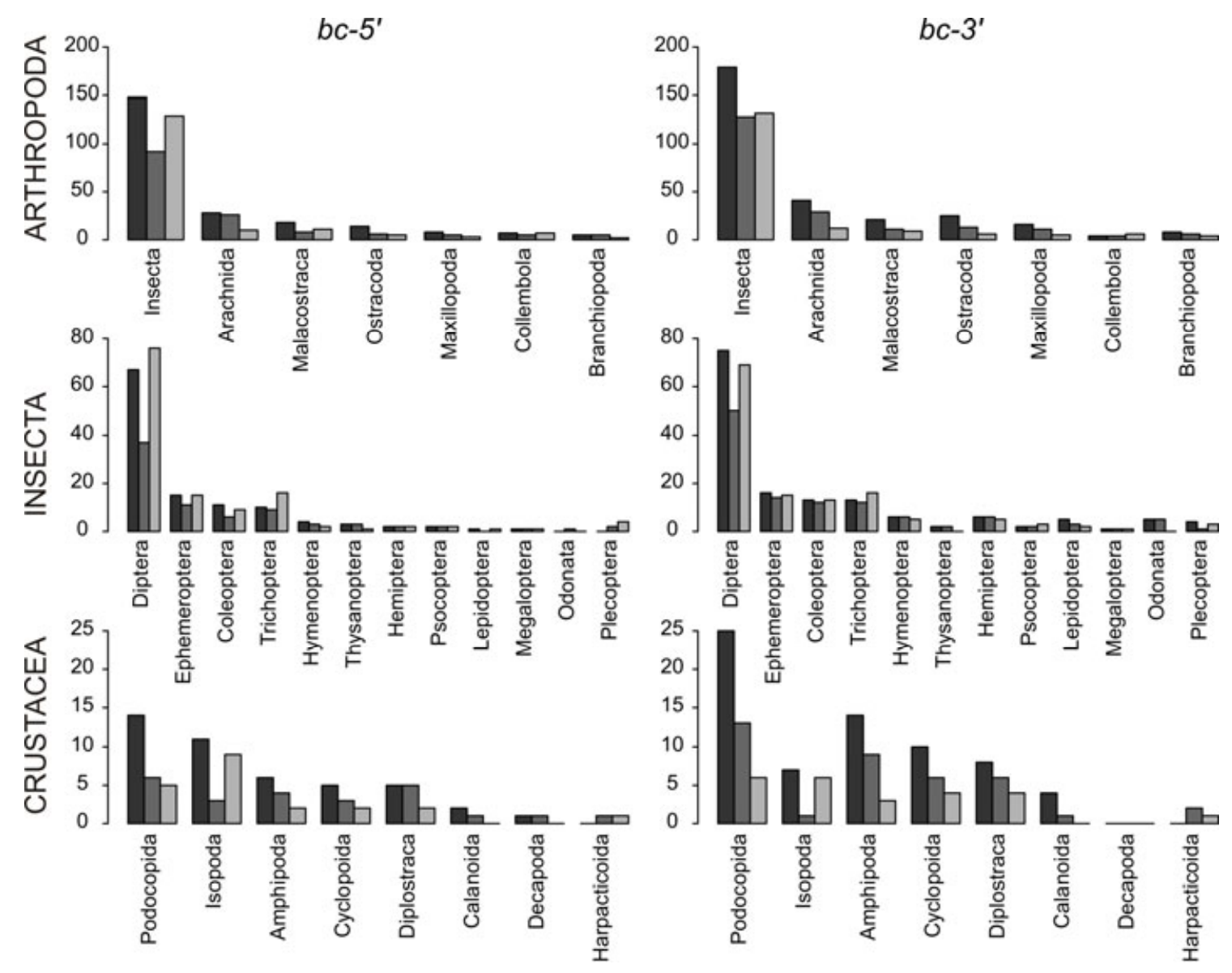

This article is protected by copyright. All rights reserved. 

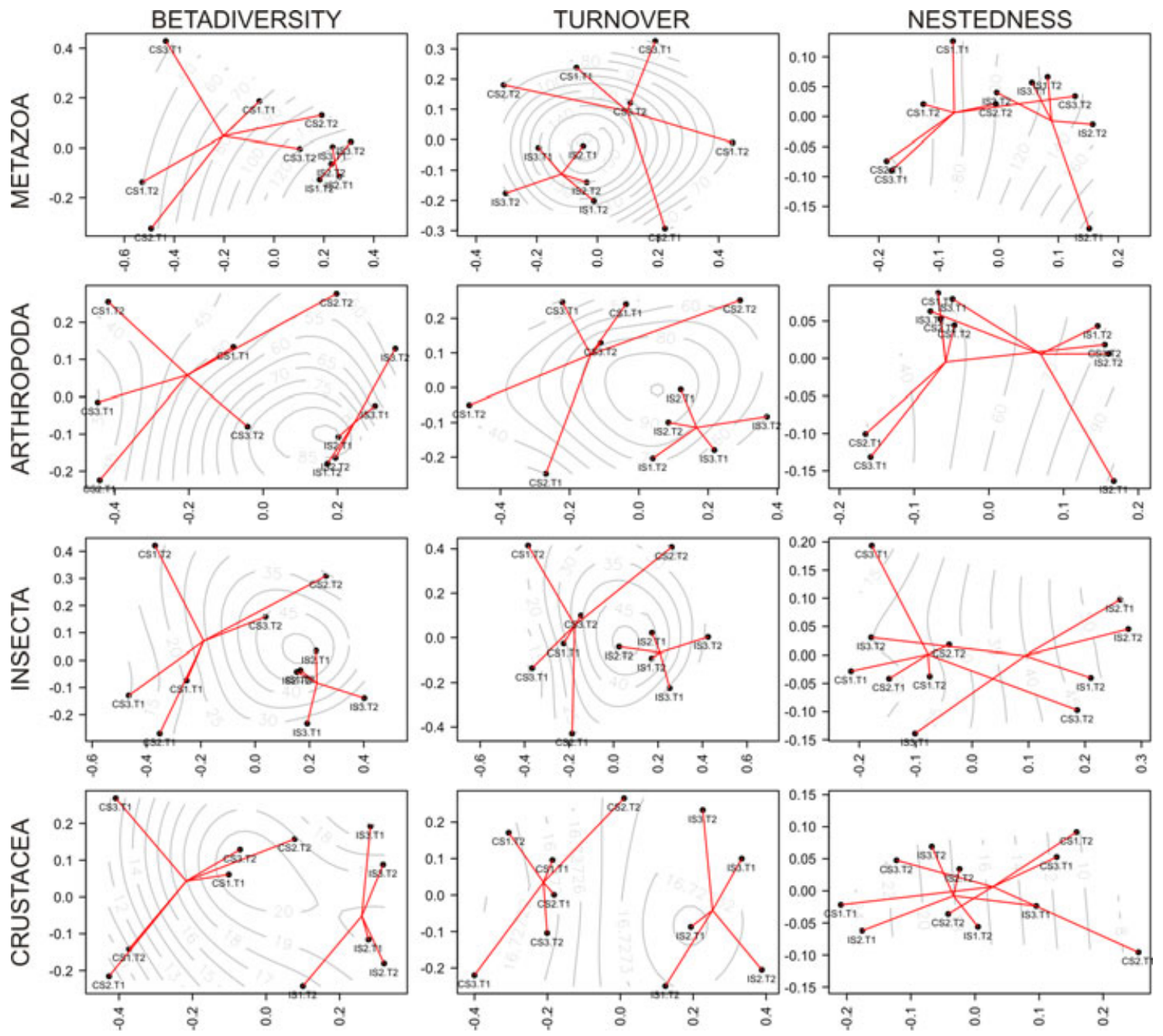

This article is protected by copyright. All rights reserved. 

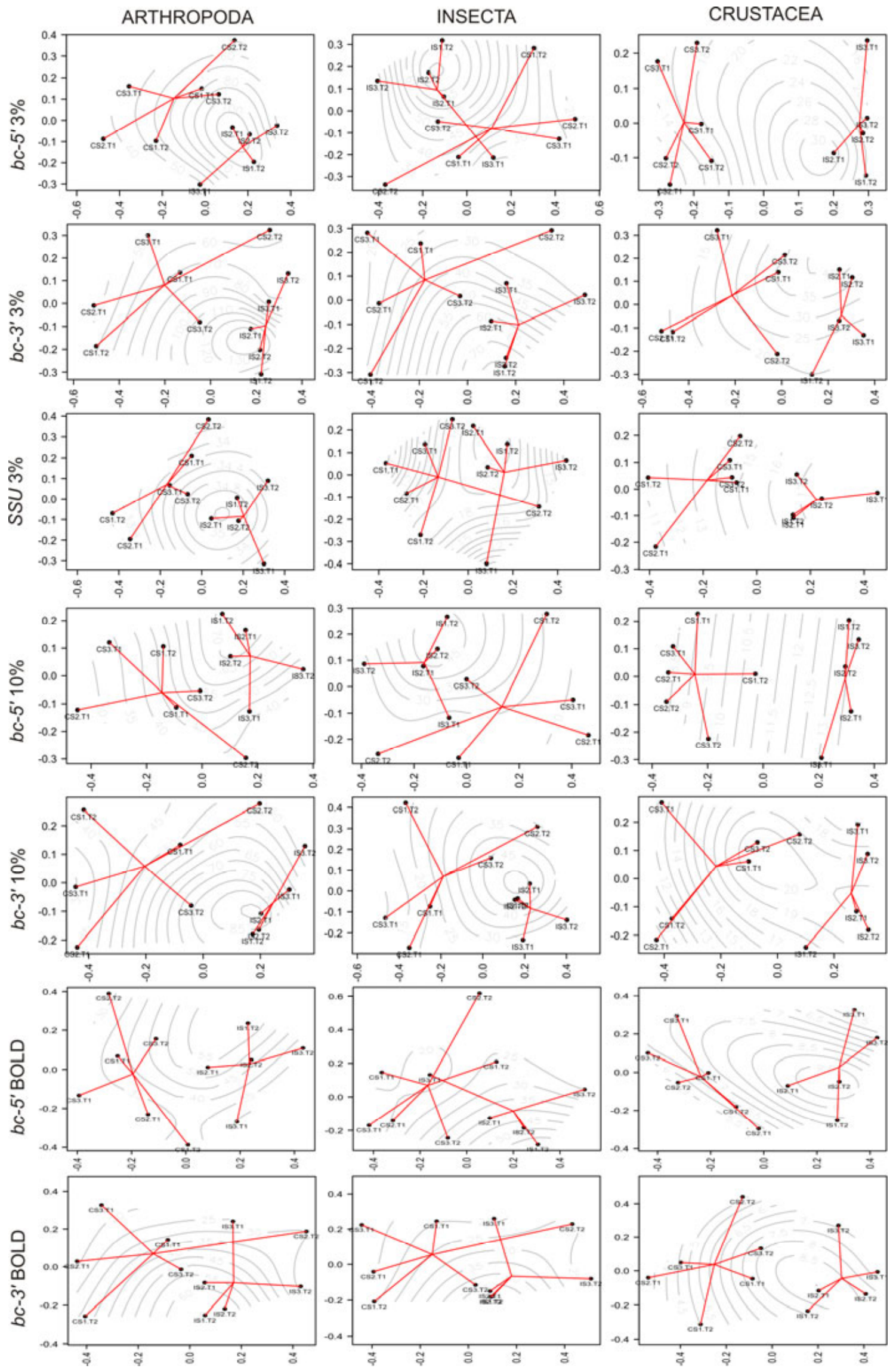

This article is protected by copyright. All rights reserved. 

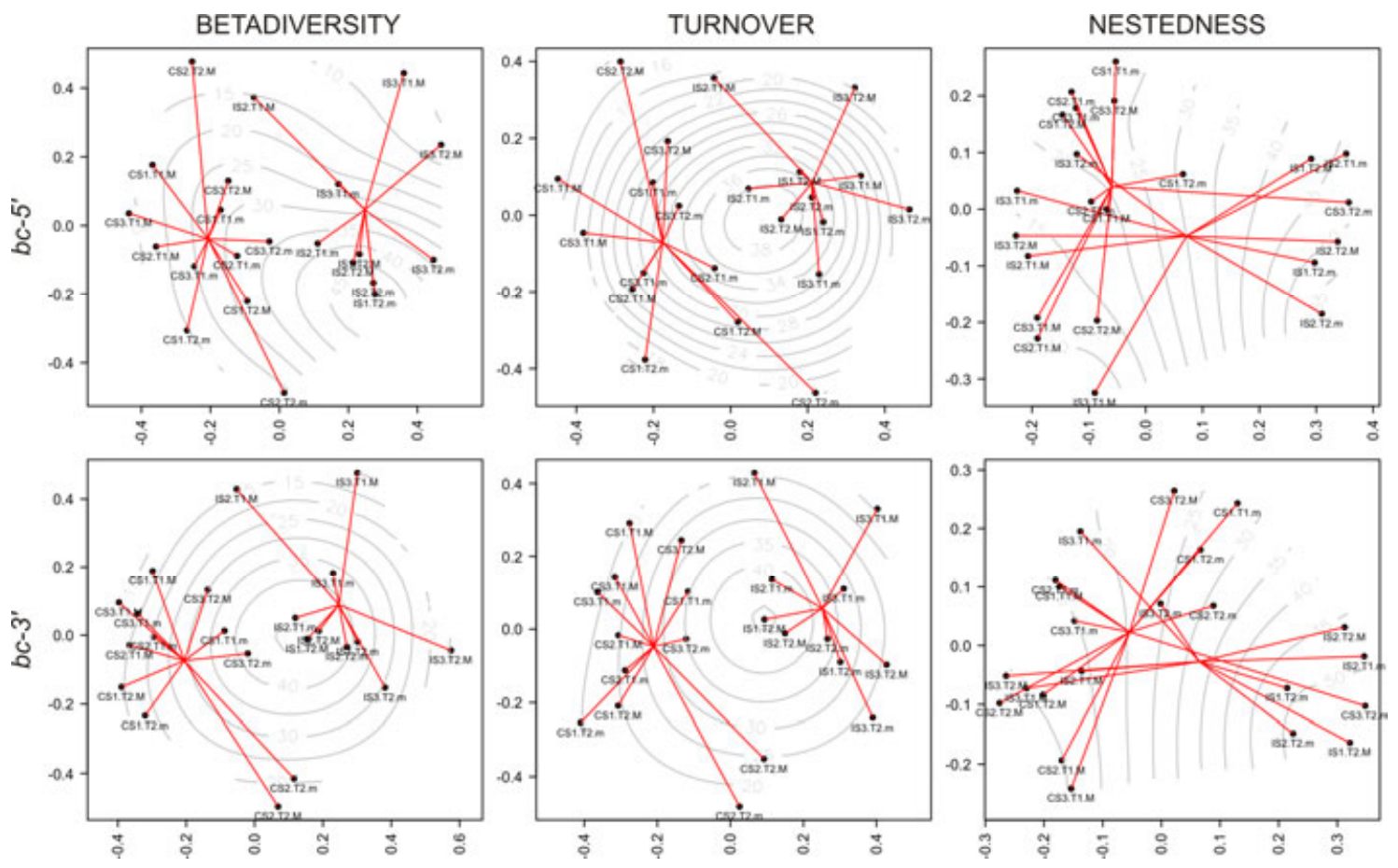

This article is protected by copyright. All rights reserved. 